Research Article

\title{
Formulation of a New Mixed Four-Node Quadrilateral Element for Static Bending Analysis of Variable Thickness Functionally Graded Material Plates
}

\author{
Pham Van Vinh (D) \\ Department of Solid Mechanics, Le Quy Don Technical University, Hanoi City 100000, Vietnam \\ Correspondence should be addressed to Pham Van Vinh; phamvanvinh@lqdtu.edu.vn
}

Received 27 November 2020; Revised 15 February 2021; Accepted 10 March 2021; Published 23 March 2021

Academic Editor: Francisco J. Martos

Copyright (c) 2021 Pham Van Vinh. This is an open access article distributed under the Creative Commons Attribution License, which permits unrestricted use, distribution, and reproduction in any medium, provided the original work is properly cited.

\begin{abstract}
A new mixed four-node quadrilateral element (MiQ4) is established in this paper to investigate functionally graded material (FGM) plates with variable thickness. The proposed element is developed based on the first-order shear deformation and mixed finite element technique, so the new element does not need any selective or reduced numerical integration. Numerous basic tests have been carried out to demonstrate the accuracy and convergence of the proposed element. Besides, the numerical examples show that the present element is free of shear locking and is insensitive to the mesh distortion, especially for the case of very thin plates. The present element can be applied to analyze plates with arbitrary geometries; it leads to reducing the computation cost. Several parameter studies are performed to show the roles of some parameters such as the power-law index, side-to-thickness ratio, boundary conditions (BCs), and variation of the plate thickness on the static bending behavior of the FGM plates.
\end{abstract}

\section{Introduction}

In the last decade of the 20th century and more than 20 years of the 21st century, functionally graded materials (FGMs) were extensively utilized in many fields of engineering as well as industry because of their advantages [1-4]. FGMs are usually produced by mixing two or more different ingredients with the volume varying continuously in the spatial direction. The most common type of FGM is ceramic-metal FGM, which is fabricated by ceramic and metal since the ceramic is compatible for working in high-temperature environments and the metal is suitable for working with the mechanical load. That is the reason why numerous researchers focus on investigating the dynamic as well as static behaviors of the FGM structures, for example, beams [5-10], plates [11-18], shells [19-21], and other structures [22, 23]. Many plate theories have been established and/or applied to investigate these structures, for example, classical plate theory (CPT), first-order shear deformation (FSDT), higherorder shear deformation theory (HSDT), and quasi-3D shear deformation theory. Thai and Choi [24] considered the static bending and free vibration of FGM plates using a simple FSDT. A simple HSDT was developed and used to examine FGM plates by Thai and Kim [25]. Zenkour [26] established a new shear deformation theory called generalized shear deformation theory (GSDT) to analyze the static bending of FGM plates. The influence of porosity on the bending of the FG plate had been studied by Hadj et al. [27] via refined plate theory. Although the FSDTs and HSDTs and their variations are suitable to predict the behavior of thin to moderate plates, it is necessary to develop quasi-3D theories to analyze thick and very thick plates. These theories take into account the normal transverse stress which is important in these types of FGM structures. Neves et al. [28] established a quasi-3D sinusoidal shear deformation theory to study the bending and free vibration of FGM plates. Thai and Kim [29] constructed a simple quasi-3D sinusoidal shear deformation theory to scrutinize FGM plates. More details on the development of the plate theory can read from these journals and their relevant references.

Although the analytical method can give exact solutions to many engineering problems, it is remarkable that it 
cannot be applied in complex problems such as analysis of complicated structures, arbitrary geometric plates, and variable thickness plates. Therefore, large numerical methods have been developed to analyze these structures. Besides, the use of the FGM structures with variable thickness is becoming the new trend in advanced structures to reduce their weight and improve the art of these constructions [30, 31], for example, the cover of the blades of the wind turbines, the integral shroud blades, and the cover of the spacecraft. These structures usually have complex geometric and boundary conditions (BCs). In these cases, the application of an analytical method to analyze the complex geometric plate is very difficult. Some worthy numerical methods can be considered herein, including differential quadrature method (DQM), generalized differential quadrature method (GDQM), finite element method (FEM), smoothed finite element method (SFEM), extended finite element method (XFEM), meshless method (MM), and isogeometric analysis (IGA). Among them, FEM is widely employed in many complicated engineering problems because of its simplification, good accuracy, and high convergence rate. It also noticed that the development of plate elements based on the Mindlin-Reissner plate theory or the FSDT has been studied for a long time and achieved a lot of success. However, at the beginning of this development, the most and major difficulty of these types of plate elements was the shear-locking phenomenon in the case of thin to very thin plates. Numerous techniques and schemes have been introduced to address this challenge. Zienkiewicz et al. [32] introduced the reduced integration technique and Hughes et al. [33] presented selective reduced integration to analyze thin to very thin plates and shells using classical four-node quadrilateral plate element (Q4). Hinton and Huang [34] developed many quadrilateral plate elements using substitute shear strain fields. Bathe and Dvorkin [35] created some plate elements by introducing the mixed interpolated tensorial components (MITC) technique, which is widely used for the analysis of thin plates and shells. A discrete shear method was developed by Batoz and Lardeur [36] for both quadrilateral and triangular plate elements. Zienkiewicz et al. [37] developed the linked interpolation technique for the Mindlin-Reissner quadrilateral plate element; then it was extended to the higher-order linked interpolation quadrilateral thick plate element by Ribaric et al. [38]. Soh et al. $[39,40]$ developed an improved shear strain interpolation technique based on Timoshenko's beam formulae. Castellazzi and Krysl [41] constructed a new displacement-based element by using the nodal integration method and assumed strain technique. Hansbo et al. [42, 43] formulated two locking-free quadrilateral elements based on continuous/ discontinuous rotations. The mixed shear projected approach element (MiSP) was established by Ayad and his colleagues $[44,45]$. The combination of the discrete shear gap technique and alternative alpha FEM was used by Nguyen-Thanh et al. [46] to analyze isotropic plates. Liu et al. $[47,48]$ developed the smoothed FEM, which integrates the strain smoothing technique into the standard FEM to construct many effective elements, such as cell-based SFEM [49-51], edge-based SFEM [52], and node-based SFEM [53].
The essential success of these elements is that they predict more accurate results, higher convergence rates, and insensitive to distorted mesh in comparison to conventional FEM. The generalized conforming elements were first developed by Long et al. [54-57] to analyze membrane, plate, and shell structures. Cen et al. [58] initiated a new element based on the quadrilateral area coordinate method for Mindlin-Reissner plates. Cen et al. [59] developed a hybridTrefftz stress element based on the hybrid displacement function (HDF) to analyze Mindlin-Reissner plates. A family of hybrid-Trefftz p-elements were established by Jirousek et al. [60] to analyze thick plates. Katili [61] used the discrete Kirchhoff-Mindlin method to establish a new plate element DKMQ to analyze thin to moderate plates. Among them, the classical Q4 element, MITC4 (four-node MITC element) element, and DKMQ element have been widely used to analyze composite and FGM plates, in which the classical Q4 element needs a reduced or selective integration and is very sensitive to the mesh distortion. The MITC4 is an efficient element with thick and moderate plates, but less accuracy with very thin plates. The DKMQ element has been developed since 1993 and it is compatible to analyze thick to thin and very thin plate, but the convergence rate is slower than MITC4 [61].

Although a lot of plate elements were developed and applied successfully for the analysis of thin to moderate plates, there are some significant challenges that remain outstanding, such as the realizable results of the element with the distorted mesh, avoiding shear locking especially in very thin plates, and good precision for the stress of the element. This paper aims to establish a new mixed fournode quadrilateral plate element based on the mixed finite element formulation and the FSDT. This is the main contribution of the current work. The displacement-based four-node element will be first used as the initiating point; then the formula of the mixed finite element will be applied to construct the proposed plate element named MiQ4 (mixed four-node quadrilateral element). Several numerical patch tests and verification studies will be considered to demonstrate the accuracy and efficiency of the proposed element MiQ4. The MiQ4 is a simple modification of the classical element Q4 so that the present element is simple in its formulation. In addition, the present element MiQ4 is free of shear locking without using reduced integration. Another advantage of the proposed element is that it is insensitive to mesh distortions, so it can be employed to analyze the FGM plates with complex geometry. Then the proposed element will be employed to research the bending behavior of the variable thickness FGM plates. A comprehensive parameter study will be carried out to demonstrate the effects of some parameters on the bending behavior of the variable thickness FGM plates.

\section{Modeling of Variable Thickness FGM Plate}

In this study, a variable thickness FGM plate with the dimension of $a \times b$ and thickness of $h$ as shown in Figure 1 is considered. The properties of the material vary continuously 


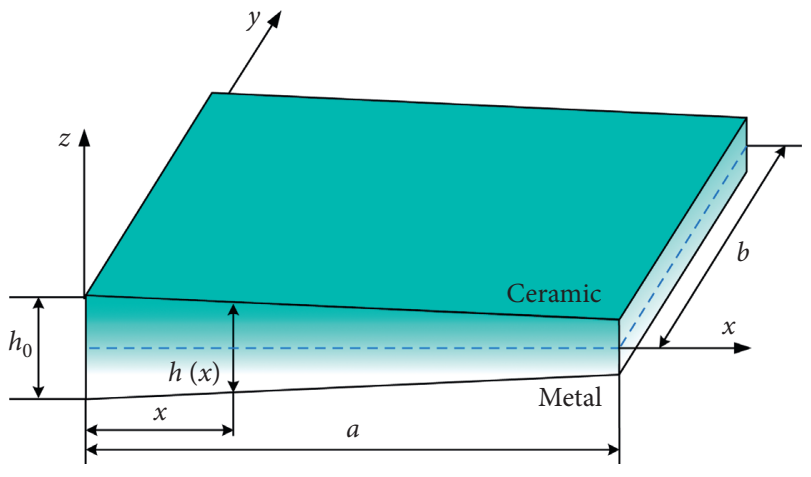

FIGURE 1: Modeling of variable thickness FGM plates.

in the thickness direction of the plates by a power-law function as the following formula $[1,14]$ :

$$
P(z)=P_{m}+\left(P_{c}-P_{m}\right)\left(\frac{2 z+h}{2 h}\right)^{p},
$$

where $P_{c}$ and $P_{m}$ are, respectively, Young's modulus $E$ or density $\rho$ of ceramic and metal and $p$ is the power-law index. In this study, the thickness of the plates $h$ depends on the variable $x$ only $h=h(x)$, and the four following types of thickness variation, which are uniform plate (U plate), linear plate (L plate), parabolic plate (P plate), and sinusoidal plate (S plate), are considered:

$$
h=h(x)= \begin{cases}h_{0}, & \text { Uniform (U plate), } \\ h_{0}\left(1-\zeta \frac{x}{a}\right), & \text { Linear ( } L \text { plate), } \\ h_{0}\left[1-\zeta\left(\frac{x}{a}\right)^{2}\right], & \text { Parabolic ( } P \text { plate }), \\ h_{0}\left[1-\zeta \sin \left(\frac{\pi x}{2 a}\right)\right], & \text { Sinusoidal (S plate) }\end{cases}
$$

where $\zeta$ is the varied parameter and $h_{0}$ is the thickness of the plate at $x=0, y=0$. In this study, the varied parameter $\zeta$ changes in the range of $0 \leq \zeta \leq 0.5$. It is noticed that the midplane of the variable thickness plate is still planar; it means that the plate is symmetric with respect to the $O x y$ plane.

\section{Governing Equations}

The displacement field of the plate based on FSDT is given as follows:

$$
\left\{\begin{array}{l}
u(x, y, z)=u(x, y)+z \psi_{x}(x, y) \\
v(x, y, z)=v(x, y)+z \psi_{y}(x, y) \\
w(x, y, z)=w(x, y)
\end{array}\right.
$$

where $u, v, w, \psi_{x}, \psi_{y}$ are five unknown displacement functions at the middle surface of the plate.
The strain field of the plates is obtained as the following formulae:

$$
\begin{aligned}
\varepsilon_{x} & =u_{, x}+z \psi_{x, x}, \\
\varepsilon_{y} & =v_{, y}+z \psi_{y, y}, \\
\gamma_{x y} & =v_{, x}+u_{, y}+z\left(\psi_{x, y}+\psi_{y, x}\right), \\
\gamma_{x z} & =w_{, x}+\psi_{x}, \\
\gamma_{y z} & =w_{, y}+\psi_{y} .
\end{aligned}
$$

The short form of equation (4) is

$$
\boldsymbol{\varepsilon}=\boldsymbol{\varepsilon}^{0}+z \boldsymbol{\varepsilon}^{1}
$$

where

$$
\begin{gathered}
\boldsymbol{\varepsilon}^{0}=\left\{\begin{array}{c}
u_{, x} \\
v_{, y} \\
u_{, y}+v_{, x}
\end{array}\right\}, \\
\boldsymbol{\varepsilon}^{1}=\left\{\begin{array}{c}
\psi_{x, x} \\
\psi_{y, y} \\
\psi_{x, y}+\psi_{y, x}
\end{array}\right\} .
\end{gathered}
$$

The vector of the transverse shear strain can be rewritten as

$$
\boldsymbol{\gamma}=\left\{\begin{array}{c}
w_{, x}+\psi_{x} \\
w_{, y}+\psi_{y}
\end{array}\right\} .
$$

The stress field of the plate is obtained as follows:

$$
\begin{aligned}
& \sigma=\mathbf{D} \varepsilon \\
& \tau=\mathbf{D}_{s} \gamma .
\end{aligned}
$$

Here,

$$
\begin{aligned}
& \mathbf{D}=\frac{E}{1-v^{2}}\left[\begin{array}{ccc}
1 & v & 0 \\
\nu & 1 & 0 \\
0 & 0 & \frac{(1-v)}{2}
\end{array}\right], \\
& \mathbf{D}_{s}=\frac{k E}{2(1+v)}\left[\begin{array}{ll}
1 & 0 \\
0 & 1
\end{array}\right],
\end{aligned}
$$

where $E$ is Young's modulus of the material and $k$ is the shear correction factor.

The strain energy of the plate and the work done by external force can be calculated as the following formulae:

$$
\begin{aligned}
\Pi_{\mathrm{st}} & =\frac{1}{2} \int_{V}\left(\boldsymbol{\varepsilon}^{T} \cdot \boldsymbol{\sigma}+\boldsymbol{\gamma}^{T} \cdot \boldsymbol{\tau}\right) \mathrm{d} V, \\
\Pi_{\mathrm{ext}} & =\int_{S} q w \mathrm{~d} S .
\end{aligned}
$$

Hamilton's principle is used to obtain the governing equation of motion of the plate 


$$
\delta \Pi=\delta \Pi_{\mathrm{st}}+\delta \Pi_{\mathrm{ext}}=0 .
$$

The variation of the strain energy is

$$
\begin{aligned}
\delta \Pi_{\text {st }} & =\frac{1}{2} \delta \int_{V}\left(\boldsymbol{\varepsilon}^{T} \boldsymbol{\sigma}+\delta \boldsymbol{\gamma}^{T} \boldsymbol{\tau}\right) \mathrm{d} V \\
& =\int_{V}\left[\delta \boldsymbol{\varepsilon}^{0}+z \delta \boldsymbol{\varepsilon}^{1}\right]^{T} \mathbf{D}\left[\delta \boldsymbol{\varepsilon}^{0}+z \delta \boldsymbol{\varepsilon}^{1}\right]+\delta \boldsymbol{\gamma}^{T} \boldsymbol{\tau} \mathrm{d} V .
\end{aligned}
$$
form:

Equation (12) can be rewritten in the following compact

$$
\delta \Pi_{\mathrm{st}}=\int_{V}\left\{\left[\delta \boldsymbol{\varepsilon}^{0} \quad \delta \boldsymbol{\varepsilon}^{1}\right]\left[\begin{array}{cc}
\mathbf{D} & z \mathbf{D} \\
z \mathbf{D} & z^{2} \mathbf{D}
\end{array}\right]\left[\begin{array}{c}
\boldsymbol{\varepsilon}^{0} \\
\boldsymbol{\varepsilon}^{1}
\end{array}\right]+\delta \boldsymbol{\gamma}^{T} \boldsymbol{\tau}\right\} \mathrm{d} V .
$$
gets

By integrating equation (13) across the thickness, one

$$
\delta \Pi_{\mathrm{st}}=\int_{S}\left(\delta \boldsymbol{\Omega}^{T} \mathbf{P}+\delta \boldsymbol{\gamma}^{T} \mathbf{Q}\right) \mathrm{d} S
$$

where $\mathbf{P}$ and $\mathbf{Q}$ are given by

$$
\begin{gathered}
\mathbf{P}=\int_{-(h(x) / 2)}^{(h(x) / 2)}\left[\begin{array}{cc}
\mathbf{D} & z \mathbf{D} \\
z \mathbf{D} & z^{2} \mathbf{D}
\end{array}\right]\left[\begin{array}{l}
\boldsymbol{\varepsilon}^{0} \\
\boldsymbol{\varepsilon}^{1}
\end{array}\right] \mathrm{d} z, \\
\mathbf{Q}=\int_{-(h(x) / 2)}^{(h(x) / 2)} \boldsymbol{\tau} \mathrm{d} z=\int_{-(h(x) / 2)}^{(h(x) / 2)} \mathbf{D}_{s} \boldsymbol{\gamma} \mathrm{d} z,
\end{gathered}
$$

and

$$
\delta \Omega=\left[\begin{array}{c}
\delta \varepsilon^{0} \\
\delta \varepsilon^{1}
\end{array}\right]
$$

Substituting equation (17) into equations (15) and (16), one gets

$$
\begin{aligned}
& \mathbf{P}=\mathbf{H} \boldsymbol{\Omega}, \\
& \mathbf{Q}=\mathbf{H}_{s} \gamma,
\end{aligned}
$$

where

$$
\begin{aligned}
\mathbf{H} & =\int_{-(h(x) / 2)}^{(h(x) / 2)}\left[\begin{array}{cc}
\mathbf{D} & z \mathbf{D} \\
z \mathbf{D} & z^{2} \mathbf{D}
\end{array}\right] \mathrm{d} z, \\
\mathbf{H}_{s} & =\int_{-(h(x) / 2)}^{(h(x) / 2)} \mathbf{D}_{s} \mathrm{~d} z .
\end{aligned}
$$

\section{Finite Element Formulation}

4.1. Displacement Finite Element Formulation. In this subsection, a brief review of a classical four-node quadrilateral element based on displacements with five degrees of freedom (DOFs) is considered as the starting point. The nodal displacement vector of the $i$-th node is

$$
\mathbf{q}_{i}=\left\{\begin{array}{lllll}
u_{i} & v_{i} & w_{i} & \psi_{x i} & \psi_{y i}
\end{array}\right\}^{T}, \quad i=\overline{1,4}
$$

The nodal displacement vector of the plate element, $\mathbf{U}$, is defined as

$$
\mathbf{U}=\left\{\begin{array}{llll}
\mathbf{q}_{1}^{T} & \mathbf{q}_{2}^{T} & \mathbf{q}_{3}^{T} & \mathbf{q}_{4}^{T}
\end{array}\right\}^{T} .
$$

Then the displacement variables at any point of the element are approximated as the following formulae:

$$
\left\{\begin{array}{l}
u=\sum N_{i} u_{i}, \\
v=\sum N_{i} v_{i}, \\
w=\sum N_{i} w_{i}, \\
\psi_{x}=\sum N_{i} \psi_{x i}, \\
\psi_{y}=\sum N_{i} \psi_{y i},
\end{array}\right.
$$

where $N_{i}$ is the bilinear shape functions of the four-node quadrilateral plate element. The expressions of the shape functions in the local coordinate are

$$
N_{i}=\frac{1}{4}\left(1+\xi_{i} \xi\right)\left(1+\eta_{i} \eta\right), \quad i=\overline{1,4} .
$$

Equation (17) can be rewritten in short form as the following formula:

$$
\delta \Omega=\mathbf{B} \delta \mathbf{U}
$$

Here,

$$
\mathbf{B}=\left[\begin{array}{llll}
\mathbf{B}^{1} & \mathbf{B}^{2} & \mathbf{B}^{3} & \mathbf{B}^{4}
\end{array}\right]
$$

with

$$
\mathbf{B}^{i}=\left[\begin{array}{ccccc}
N_{i, x} & 0 & 0 & 0 & 0 \\
0 & N_{i, y} & 0 & 0 & 0 \\
N_{i, y} & N_{i, x} & 0 & 0 & 0 \\
0 & 0 & 0 & N_{i, x} & 0 \\
0 & 0 & 0 & 0 & N_{i, y} \\
0 & 0 & 0 & N_{i, y} & N_{i, x}
\end{array}\right], \quad i=\overline{1,4} .
$$

The variation of the shear strain vector is obtained as follows:

$$
\delta \boldsymbol{\gamma}=\left\{\begin{array}{c}
\delta w_{, x}+\delta \psi_{x} \\
\delta w_{, y}+\delta \psi_{y}
\end{array}\right\}
$$

Equation (28) can be written in short form as

$$
\delta \gamma=\mathbf{B}_{s} \delta \mathbf{U}
$$

where

$$
\mathbf{B}_{s}=\left[\begin{array}{llll}
\mathbf{B}_{s}^{1} & \mathbf{B}_{s}^{2} & \mathbf{B}_{s}^{3} & \mathbf{B}_{s}^{4}
\end{array}\right],
$$

with

$$
\mathbf{B}_{s}^{i}=\left[\begin{array}{ccccc}
0 & 0 & N_{i, x} & N_{i} & 0 \\
0 & 0 & N_{i, y} & 0 & N_{i}
\end{array}\right], \quad i=\overline{1,4} .
$$


By substituting equations (25) and (29) into the expression of the variation of the strain energy, we get

$$
\delta \Pi_{\mathrm{st}}=\int_{S} \delta \mathbf{U}^{T} \mathbf{B}^{T} \mathbf{H B U d} S+\int_{S} \delta \mathbf{U}^{T} \mathbf{B}_{s}^{T} \mathbf{H}_{s} \mathbf{B}_{s} \mathbf{U d} S .
$$

The variation of the work done by an external force is calculated by

$$
\delta \Pi_{\mathrm{ext}}=-\int_{S} q \delta w \mathrm{~d} S=-\delta \mathbf{U}^{T} \int_{S} \mathbf{N}_{w}^{T} q \mathrm{~d} S .
$$

Here, $\mathbf{N}_{w}$ is the matrix of the shape functions related to the transverse displacement; its expression is as the following formula:

$$
\mathbf{N}_{w}=\left[\begin{array}{llll}
\mathbf{N}_{w 1} & \mathbf{N}_{w 2} & \mathbf{N}_{w 3} & \mathbf{N}_{w 4}
\end{array}\right]
$$

where

$$
\mathbf{N}_{w i}=\left[\begin{array}{lllll}
0 & 0 & N_{i} & 0 & 0
\end{array}\right], \quad i=\overline{1,4} .
$$

Inserting equations (32) and (33) into equation (11) and using the trivial manner of classical FEM, one gets

$$
\mathbf{K U}=\mathbf{f} .
$$

Here, the stiffness matrix and the nodal force vector of the plate element are computed by the following formulae:

$$
\mathbf{K}=\mathbf{K}_{b}+\mathbf{K}_{s} \text {, }
$$

where

$$
\begin{aligned}
\mathbf{K}_{b} & =\int_{S} \mathbf{B}^{T} \mathbf{H B} \mathrm{d} S=\int_{-1}^{1} \int_{-1}^{1} \mathbf{B}^{T} \mathbf{H B}|\mathbf{J}| \mathrm{d} \xi \mathrm{d} \eta \\
\mathbf{K}_{s} & =\int_{S} \mathbf{B}_{s}^{T} \mathbf{H}_{s} \mathbf{B}_{s} \mathrm{~d} S=\int_{-1}^{1} \int_{-1}^{1} \mathbf{B}_{s}^{T} \mathbf{H}_{s} \mathbf{B}_{s}|\mathbf{J}| \mathrm{d} \xi \mathrm{d} \eta, \\
\mathbf{f} & =\int_{S} \mathbf{N}_{w}^{T} q \mathrm{~d} S=\int_{-1}^{1} \int_{-1}^{1} \mathbf{N}_{w}^{T} q|\mathbf{J}| \mathrm{d} \xi \mathrm{d} \eta .
\end{aligned}
$$

The Gauss integration is used to estimate the stiffness matrix $\mathbf{K}_{b}, \mathbf{K}_{s}$ as well as the nodal force vector $\mathbf{f}$. For each point of Gauss integration, the integration through the thickness direction of equations (19) and (20) will be evaluated via Newton-Cotes quadrature rules. It is noticed that $\mathbf{K}_{b}$ and $\mathbf{f}$ are calculated by using full Gauss integration $(2 \times 2)$, while the shear stiffness matrix $\mathbf{K}_{s}$ is estimated by using reduced Gauss integration $(1 \times 1)$ to avoid the wellknown shear-locking phenomenon. In this study, the acronym Q4 is used to denote the classical four-node quadrilateral plate element based on the displacement formula with reduced Gauss integration.

4.2. Mixed Finite Element Formulation. In this subsection, we introduce a quadratic interpolation for $\psi_{x}, \psi_{y}$, and then the expressions of $\psi_{x}, \psi_{y}$ are obtained as follows:

$$
\left\{\begin{array}{l}
\psi_{x}=\sum N_{i} \psi_{x i}+N_{m} \psi_{x m} \\
\psi_{y}=\sum N_{i} \psi_{y i}+N_{m} \psi_{y m}
\end{array}\right.
$$

where

$$
N_{m}=\frac{1}{16}\left(1-\xi^{2}\right)\left(1-\eta^{2}\right)
$$

Substituting equation (39) into equation (25), the expression of $\delta \varepsilon^{1}$ can be obtained as follows:

$$
\begin{aligned}
\delta \boldsymbol{\varepsilon}^{1}= & \left\{\begin{array}{c}
\sum N_{i, x} \delta \psi_{x i} \\
\sum N_{i, y} \delta \psi_{y i} \\
\sum N_{i, y} \delta \psi_{x i}+\sum N_{i, x} \delta \psi_{y i}
\end{array}\right\} \\
& +\left\{\begin{array}{c}
N_{m, x} \delta \psi_{x m} \\
N_{m, y} \delta \psi_{y m} \\
N_{m, y} \delta \psi_{x m}+N_{m, x} \delta \psi_{y m}
\end{array}\right\},
\end{aligned}
$$

or

$$
\delta \boldsymbol{\varepsilon}^{1}=\left\{\begin{array}{c}
\sum N_{i, x} \delta \psi_{x i} \\
\sum N_{i, y} \delta \psi_{y i} \\
\sum N_{i, y} \delta \psi_{x i}+\sum N_{i, x} \delta \psi_{y i}
\end{array}\right\}+\left[\begin{array}{cc}
N_{m, x} & 0 \\
0 & N_{m, y} \\
N_{m, y} & N_{m, x}
\end{array}\right]\left\{\begin{array}{c}
\delta \psi_{x m} \\
\delta \psi_{y m}
\end{array}\right\} .
$$

The expression of the strain vector is obtained as follows:

$$
\delta \boldsymbol{\Omega}=\mathbf{B} \delta \mathbf{U}+\mathbf{B}_{m} \delta \boldsymbol{\psi}_{m},
$$

where

$$
\begin{gathered}
\mathbf{B}_{m}=\left[\begin{array}{cc}
\text { 0 } \\
N_{m, x} & 0 \\
0 & N_{m, y} \\
N_{m, y} & N_{m, x}
\end{array}\right], \\
\delta \psi_{m}=\left\{\begin{array}{c}
\delta \psi_{x m} \\
\delta \psi_{y m}
\end{array}\right\} .
\end{gathered}
$$

The shear strain $\delta \gamma$ is expressed as the following formula:

$$
\delta \gamma=\left\{\begin{array}{c}
\sum N_{i, x} \delta w_{i}+\sum N_{i} \delta \psi_{x i}+N_{m} \delta \psi_{x m} \\
\sum N_{i, y} \delta w_{i}+\sum N_{i} \delta \psi_{y i}+N_{m} \delta \psi_{y m}
\end{array}\right\}
$$

or

$$
\delta \boldsymbol{\gamma}=\left\{\begin{array}{l}
\sum N_{i, x} \delta w_{i}+\sum N_{i} \delta \psi_{x i} \\
\sum N_{i, y} \delta w_{i}+\sum N_{i} \delta \psi_{y i}
\end{array}\right\}+\left[\begin{array}{cc}
N_{m} & 0 \\
0 & N_{m}
\end{array}\right]\left\{\begin{array}{l}
\delta \psi_{x m} \\
\delta \psi_{y m}
\end{array}\right\} .
$$

We rewrite equation (46) in the matrix form as follows:

$$
\delta \boldsymbol{\gamma}=\mathbf{B}_{s} \delta \mathbf{U}+\mathbf{M}_{m} \delta \psi_{m}
$$

where 


$$
\begin{aligned}
\mathbf{M}_{m} & =\left[\begin{array}{cc}
N_{m} & 0 \\
0 & N_{m}
\end{array}\right], \\
\delta \boldsymbol{\psi}_{m} & =\left\{\begin{array}{l}
\delta \psi_{x m} \\
\delta \psi_{y m}
\end{array}\right\} .
\end{aligned}
$$

We introduce a constant shear strain field $\bar{\gamma}=\mathbf{H}_{s}^{-1} \mathbf{Q}_{0}$, and following the procedure of the mixed FEM, the expression of the potential energy is evaluated as [62]

$\Pi=\frac{1}{2} \int_{S}\left(\boldsymbol{\Omega}^{T} \mathbf{H} \boldsymbol{\Omega}+\boldsymbol{\gamma}^{T} \mathbf{H}_{s} \bar{\gamma}+\bar{\gamma}^{T} \mathbf{H}_{s} \boldsymbol{\gamma}-\bar{\gamma}^{T} \mathbf{H}_{s} \bar{\gamma}\right) \mathrm{d} S+\Pi_{\mathrm{ext}}+\Pi_{\mathrm{bc}}$, where $\Pi_{b c}$ is the energy of the constrain to consider the BC effects.

The variations of equation (49) are

$$
\begin{array}{r}
\int_{S}\left(\delta \boldsymbol{\Omega}^{T} \mathbf{H} \boldsymbol{\Omega}+\delta \boldsymbol{\gamma}^{T} \mathbf{H}_{s} \bar{\gamma}\right) \mathrm{d} S-\delta \Pi_{\mathrm{ext}}=0, \\
\int_{S} \delta \bar{\gamma}^{T} \mathbf{H}_{s}(\boldsymbol{\gamma}-\bar{\gamma}) \mathrm{d} S=0 .
\end{array}
$$

Inserting equations (43) and (47) into equations (50) and (51), one gets

$$
\begin{array}{r}
\int_{S}\left(\left(\mathbf{B} \delta \mathbf{U}+\mathbf{B}_{m} \delta \boldsymbol{\psi}_{m}\right)^{T} \mathbf{H}\left(\mathbf{B U}+\mathbf{B}_{m} \boldsymbol{\psi}_{m}\right)+\left(\mathbf{B}_{s} \delta \mathbf{U}+\mathbf{M}_{m} \delta \boldsymbol{\psi}_{m}\right)^{T} \mathbf{H}_{s} \bar{\gamma}\right) \mathrm{d} S+\delta \Pi_{\mathrm{ext}}=0, \\
\int_{S} \delta \bar{\gamma}^{T} \mathbf{H}_{s}\left(\left(\mathbf{B}_{s} \mathbf{U}+\mathbf{M}_{m} \boldsymbol{\psi}_{m}\right)-\bar{\gamma}\right) \mathrm{d} S=\mathbf{0} .
\end{array}
$$

Inserting $\bar{\gamma}=\mathbf{H}_{s}^{-1} \mathbf{Q}_{0}$ into equations (52) and (53), one gets

$$
\begin{array}{r}
\int_{S}\left(\left(\mathbf{B} \delta \mathbf{U}+\mathbf{B}_{m} \delta \boldsymbol{\psi}_{m}\right)^{T} \mathbf{H}\left(\mathbf{B} \mathbf{U}+\mathbf{B}_{m} \boldsymbol{\psi}_{m}\right)+\left(\mathbf{B}_{s} \delta \mathbf{U}+\mathbf{M}_{m} \delta \boldsymbol{\psi}_{m}\right)^{T} \mathbf{Q}_{0}\right) \mathrm{d} S+\delta \Pi_{\mathrm{ext}}=0 \\
\int_{S} \delta \mathbf{T}_{0}^{T}\left(\left(\mathbf{B}_{s} \mathbf{U}+\mathbf{M}_{m} \boldsymbol{\psi}_{m}\right)-\mathbf{H}_{s}^{-1} \mathbf{Q}_{0}\right) \mathrm{d} S=\mathbf{0}
\end{array}
$$

or

$$
\begin{aligned}
\int_{S}\left(\delta \mathbf{U}^{T} \mathbf{B}^{T} \mathbf{H B U}+\delta \mathbf{U}^{T} \mathbf{B}^{T} \mathbf{H} \mathbf{B}_{m} \boldsymbol{\psi}_{m}+\delta \boldsymbol{\psi}_{m}^{T} \mathbf{B}_{m}^{T} \mathbf{H B U}+\delta \boldsymbol{\psi}_{m}^{T} \mathbf{B}_{m}^{T} \mathbf{H} \mathbf{B}_{m} \boldsymbol{\psi}_{m}+\delta \mathbf{U}^{T} \mathbf{B}_{s}^{T} \mathbf{Q}_{0}+\delta \boldsymbol{\psi}_{m}^{T} \mathbf{M}_{m}^{T} \mathbf{Q}_{0}\right) \mathrm{d} S-\delta \mathbf{U}^{T} \int_{S} \mathbf{N}_{w}^{T} q \mathrm{~d} S=0 \\
\qquad \int_{S}\left(\delta \mathbf{Q}_{0}^{T} \mathbf{B}_{s} \mathbf{U}+\delta \mathbf{Q}_{0}^{T} \mathbf{M}_{m} \boldsymbol{\psi}_{m}+\delta \mathbf{Q}_{0}^{T}\left(-\mathbf{H}_{s}^{-1}\right) \mathbf{Q}_{0}\right) \mathrm{d} S=\mathbf{0}
\end{aligned}
$$

By combining equations (53) and (57), one gets

$$
\left[\begin{array}{lll}
\delta \mathbf{U}^{T} & \delta \mathbf{Q}_{0}^{T} & \delta \boldsymbol{\psi}_{m}^{T}
\end{array}\right]\left(\left[\begin{array}{ccc}
\mathbf{K}_{u u} & \mathbf{K}_{u T} & \mathbf{K}_{u \psi} \\
\mathbf{K}_{u T}^{T} & \mathbf{K}_{T T} & \mathbf{K}_{T \psi} \\
\mathbf{K}_{u \psi}^{T} & \mathbf{K}_{T \psi}^{T} & \mathbf{K}_{\psi \psi}
\end{array}\right]\left[\begin{array}{c}
\mathbf{U} \\
\mathbf{Q}_{0} \\
\boldsymbol{\psi}_{m}
\end{array}\right]-\left[\begin{array}{c}
\mathbf{f}_{u} \\
\mathbf{0} \\
\mathbf{0}
\end{array}\right]\right)=0,
$$

where 


$$
\begin{aligned}
& \mathbf{K}_{u u}=\int_{S} \mathbf{B}^{T} \mathbf{H B} \mathrm{d} S=\int_{-1}^{1} \int_{-1}^{1} \mathbf{B}^{T} \mathbf{H B}|\mathbf{J}| d \xi \mathrm{d} \eta, \\
& \mathbf{K}_{u \psi}=\int_{S} \mathbf{B}^{T} \mathbf{H B}_{m} \mathrm{~d} S=\int_{-1}^{1} \int_{-1}^{1} \mathbf{B}^{T} \mathbf{H} \mathbf{B}_{m}|\mathbf{J}| \mathrm{d} \xi \mathrm{d} \eta \\
& \mathbf{K}_{\psi \psi}=\int_{S} \mathbf{B}_{m}^{T} \mathbf{H} \mathbf{B}_{m} \mathrm{~d} S=\int_{-1}^{1} \int_{-1}^{1} \mathbf{B}_{m}^{T} \mathbf{H} \mathbf{B}_{m}|\mathbf{J}| \mathrm{d} \xi \mathrm{d} \eta . \\
& \mathbf{K}_{u T}=\int_{S} \mathbf{B}_{s}^{T} \mathrm{~d} S=\int_{-1}^{1} \int_{-1}^{1} \mathbf{B}_{s}^{T}|\mathbf{J}| \mathrm{d} \xi \mathrm{d} \eta, \\
& \mathbf{K}_{T \psi}=\int_{S} \mathbf{M}_{m} \mathrm{~d} S=\int_{-1}^{1} \int_{-1}^{1} \mathbf{M}_{m}|\mathbf{J}| \mathrm{d} \xi \mathrm{d} \eta, \\
& \mathbf{K}_{T T}=-\int_{S} \mathbf{H}_{s}^{-1} \mathrm{~d} S=-\int_{-1}^{1} \int_{-1}^{1} \mathbf{H}_{s}^{-1}|\mathbf{J}| \mathrm{d} \xi \mathrm{d} \eta, \\
& \mathbf{f}_{u}=\int_{S} \mathbf{N}_{w}^{T} q \mathrm{~d} S=\int_{-1}^{1} \int_{-1}^{1} \mathbf{N}_{w}^{T} q|\mathbf{J}| \mathrm{d} \xi \mathrm{d} \eta
\end{aligned}
$$

where $|\mathbf{J}|$ is the determinant of the Jacobian matrix.

By eliminating $\psi_{m}$ and $\mathbf{Q}_{0}$ from equation (58) at the element level, one gets

$$
\mathbf{K U}=\mathbf{f}
$$

in which

$$
\begin{aligned}
& \mathbf{K}=\mathbf{K}_{11}-\mathbf{K}_{12} \mathbf{K}_{22}^{-1} \mathbf{K}_{21}, \\
& \mathbf{f}=\mathbf{f}_{\mathcal{u}},
\end{aligned}
$$

where

$$
\begin{aligned}
& \mathbf{K}_{11}=\mathbf{K}_{u u}-\mathbf{K}_{u \psi} \mathbf{K}_{\psi \psi}^{-1} \mathbf{K}_{u \psi}^{T}, \\
& \mathbf{K}_{12}=\mathbf{K}_{u T}-\mathbf{K}_{u \psi} \mathbf{K}_{\psi \psi}^{-1} \mathbf{K}_{T \psi}^{T}, \\
& \mathbf{K}_{21}=\mathbf{K}_{u T}^{T}-\mathbf{K}_{T \psi} \mathbf{K}_{\psi \psi}^{-1} \mathbf{K}_{u \psi}^{T}, \\
& \mathbf{K}_{22}=\mathbf{K}_{T T}-\mathbf{K}_{T \psi} \mathbf{K}_{\psi \psi}^{-1} \mathbf{K}_{T \psi}^{T} .
\end{aligned}
$$

For convenience, the acronym MiQ4 is used to denote the proposed mixed four-node quadrilateral plate element. It is noticed that all integrations in equations (59)-(65) relate to the element stiffness matrix, the nodal force vector, and they are calculated using full Gauss integration, but the shear-locking phenomenon does not appear as we will appreciate and discuss in the next section. Besides, the proposed plate element MiQ4 is insensitive to mesh distortion, so it can be employed to analyze the plates without worry about the complicated geometries. Moreover, the shape functions of MiQ4 are bilinear and the number of DOFs of MiQ4 is equal to that of Q4, so the computation cost is reduced. They are substantial benefits of the proposed plate element in comparison with the classical four-node quadrilateral element Q4 and other four-node quadrilateral elements.

\section{Numerical Results and Discussions}

5.1. Convergence Study. In this subsection, the convergence studies are considered for square isotropic plates subjected to uniform load as shown in Figure 2. The convergences of the proposed element MiQ4 with full Gauss integration are compared with those of classical Q4 with reduced Gauss integration. The nondimensional central deflections of reference $\left(w_{\text {ref }} .100 \mathrm{D} / \mathrm{qa}^{4}\right)$ in this subsection are calculated using the formulation given by Jirousek et al. [60]. The dimension and material properties of the plate are $a=10$, $E=10.92$, and $\nu=0.3$ and the thickness-to-side ratio $h / a$ of the plates is $0.1,0.01$, and 0.001 , respectively. The plates are divided by a regular rectangular mesh of $N \times N$.

The comparisons of nondimensional central deflection $\left(w_{c} .100 \mathrm{D} / \mathrm{qa}^{4}\right)$ of the plates using MiQ4 and Q4 and reference results are presented in Tables 1-3, in which the error of the numerical results is computed in comparison to the solutions of Jirousek et al. [60]. The errors of the numerical results using MiQ4 and Q4 and reference results with different meshes and BCs are displayed in Figure 3. According to Tables 1-3 and Figure 3, it can be seen that the proposed element MiQ4 is an excellent convergence speed for the case of fully simple supported (SSSS) plates and good convergence speed for the case of fully clamped (CCCC) ones. In comparison with Q4, although the MiQ4 element is using full Gauss integration for all its integral formulae of stiffness matrix and nodal force vector, the convergence rate of MiQ4 is better than the Q4 element, especially for coarse meshes and very thin plates. Besides, the proposed element MiQ4 seems stiffer than the classical element Q4. In the case of SSSS plates, the convergence of the MiQ4 element is better than that of the Q4 element for thick to thin plates. In the case of CCCC plates, the convergence of the MiQ4 element is worse than that of the Q4 element; this is a disadvantage of the proposed element. However, the element MiQ4 is much insensitive with mesh distortion which will be demonstrated in the following subsections.

Additionally, a benchmark test about the shear locking free of the proposed plate element MiQ4 is presented. The geometrical and material properties of the plate are retained while the thickness-to-side ratio changes from the thin case $\left(h / a=10^{-3}\right)$ to the very thin case $\left(h / a=10^{-30}\right)$. The numerical results of the classical Q4 element with reduced integration, the proposed element MiQ4 with full integration, HDF element HDF $-P 4-11 \beta$ of Cen et al. [59], and analytical solution [59] are given in Table 4. It is obvious that the proposed element is free of shear locking even in very thin plates, while the classical Q4 element cannot work 


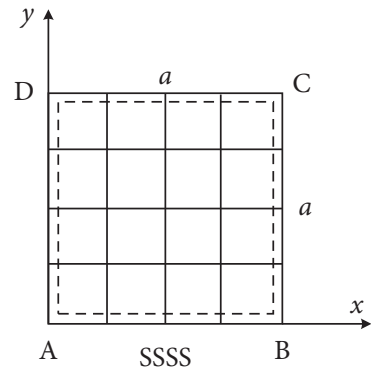

(a)

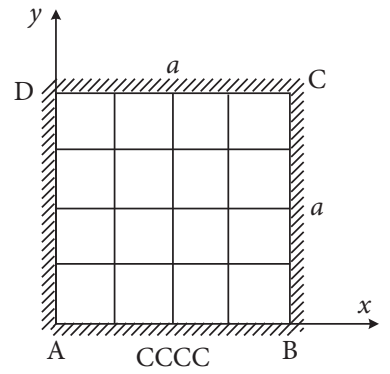

(b)

FIgURE 2: Model of square isotropic plates.

TABle 1: Nondimensional central deflection $\left(w_{c} .100 \mathrm{D} / \mathrm{qa}^{4}\right)$ of the square plate with $h / a=0.1$.

\begin{tabular}{|c|c|c|c|c|c|c|c|c|}
\hline \multirow{2}{*}{ Mesh } & \multicolumn{4}{|c|}{ SSSS } & \multicolumn{4}{|c|}{ CCCC } \\
\hline & Q4 & Error (\%) & MiQ4 & Error (\%) & Q4 & Error (\%) & MiQ4 & Error $(\%)$ \\
\hline $2 \times 2$ & 0.354582 & -17.01 & 0.463333 & 8.44 & 0.035714 & -76.26 & 0.228609 & 51.94 \\
\hline $4 \times 4$ & 0.420696 & -1.54 & 0.434501 & 1.69 & 0.145856 & -3.06 & 0.171408 & 13.92 \\
\hline $6 \times 6$ & 0.424538 & -0.64 & 0.430072 & 0.65 & 0.148648 & -1.20 & 0.159182 & 5.80 \\
\hline $8 \times 8$ & 0.425739 & -0.36 & 0.428772 & 0.35 & 0.149469 & -0.66 & 0.155345 & 3.25 \\
\hline $10 \times 10$ & 0.426292 & -0.23 & 0.428212 & 0.22 & 0.149828 & -0.42 & 0.153564 & 2.06 \\
\hline $12 \times 12$ & 0.426592 & -0.16 & 0.427918 & 0.15 & 0.150021 & -0.29 & 0.152607 & 1.43 \\
\hline $14 \times 14$ & 0.426773 & -0.12 & 0.427744 & 0.11 & 0.150137 & -0.21 & 0.152033 & 1.05 \\
\hline $16 \times 16$ & 0.426890 & -0.09 & 0.427632 & 0.08 & 0.150212 & -0.16 & 0.151662 & 0.80 \\
\hline$[60]$ & \multicolumn{4}{|c|}{0.427284} & \multicolumn{4}{|c|}{0.150460} \\
\hline
\end{tabular}

TABLE 2: Central deflection $\left(w_{c} .100 \mathrm{D} / \mathrm{qa}^{4}\right)$ of the square plate with $h / a=0.01$.

\begin{tabular}{|c|c|c|c|c|c|c|c|c|}
\hline \multirow{2}{*}{ Mesh } & \multicolumn{4}{|c|}{ SSSS } & \multicolumn{4}{|c|}{ CCCC } \\
\hline & Q4 & Error (\%) & MiQ4 & Error (\%) & Q4 & Error (\%) & MiQ4 & Error (\%) \\
\hline $2 \times 2$ & 0.319225 & -21.46 & 0.427976 & 5.30 & 0.000357 & -99.72 & 0.193253 & 53.38 \\
\hline $4 \times 4$ & 0.397125 & -2.29 & 0.410966 & 1.11 & 0.121379 & -3.67 & 0.146045 & 15.91 \\
\hline $6 \times 6$ & 0.402650 & -0.93 & 0.408192 & 0.43 & 0.124209 & -1.42 & 0.135131 & 7.25 \\
\hline $8 \times 8$ & 0.404346 & -0.52 & 0.407381 & 0.23 & 0.125326 & -0.53 & 0.131496 & 4.36 \\
\hline $10 \times 10$ & 0.405109 & -0.33 & 0.407030 & 0.14 & 0.125850 & -0.12 & 0.129785 & 3.00 \\
\hline $12 \times 12$ & 0.405518 & -0.23 & 0.406844 & 0.10 & 0.126134 & 0.11 & 0.128865 & 2.27 \\
\hline $14 \times 14$ & 0.405763 & -0.17 & 0.406734 & 0.07 & 0.126305 & 0.24 & 0.128310 & 1.83 \\
\hline $16 \times 16$ & 0.405921 & -0.13 & 0.406663 & 0.05 & 0.126417 & 0.33 & 0.127951 & 1.55 \\
\hline$[60]$ & \multicolumn{4}{|c|}{0.406446} & \multicolumn{4}{|c|}{0.12600} \\
\hline
\end{tabular}

TABLe 3: Nondimensional central deflection $\left(w_{c} .100 \mathrm{D} / \mathrm{qa}^{4}\right)$ of the square plate with $h / a=0.001$.

\begin{tabular}{|c|c|c|c|c|c|c|c|c|}
\hline \multirow{2}{*}{ Mesh } & \multicolumn{4}{|c|}{ SSSS } & \multicolumn{4}{|c|}{ CCCC } \\
\hline & Q4 & Error (\%) & MiQ4 & Error (\%) & Q4 & Error (\%) & MiQ4 & Error (\%) \\
\hline $2 \times 2$ & 0.318872 & -21.51 & 0.427622 & 5.26 & 0.000004 & -100.00 & 0.192899 & 53.09 \\
\hline $4 \times 4$ & 0.396889 & -2.30 & 0.410731 & 1.11 & 0.121123 & -3.87 & 0.145783 & 15.70 \\
\hline $6 \times 6$ & 0.402431 & -0.94 & 0.407974 & 0.43 & 0.123950 & -1.63 & 0.134883 & 7.05 \\
\hline $8 \times 8$ & 0.404132 & -0.52 & 0.407168 & 0.23 & 0.125068 & -0.74 & 0.131246 & 4.16 \\
\hline $10 \times 10$ & 0.404897 & -0.33 & 0.406818 & 0.14 & 0.125593 & -0.32 & 0.129535 & 2.81 \\
\hline $12 \times 12$ & 0.405307 & -0.23 & 0.406634 & 0.10 & 0.125879 & -0.10 & 0.128615 & 2.08 \\
\hline $14 \times 14$ & 0.405553 & -0.17 & 0.406524 & 0.07 & 0.126051 & 0.04 & 0.128060 & 1.63 \\
\hline $16 \times 16$ & 0.405711 & -0.13 & 0.406453 & 0.05 & 0.126163 & 0.13 & 0.127701 & 1.35 \\
\hline$[60]$ & \multicolumn{4}{|c|}{0.406237} & \multicolumn{4}{|c|}{0.12600} \\
\hline
\end{tabular}




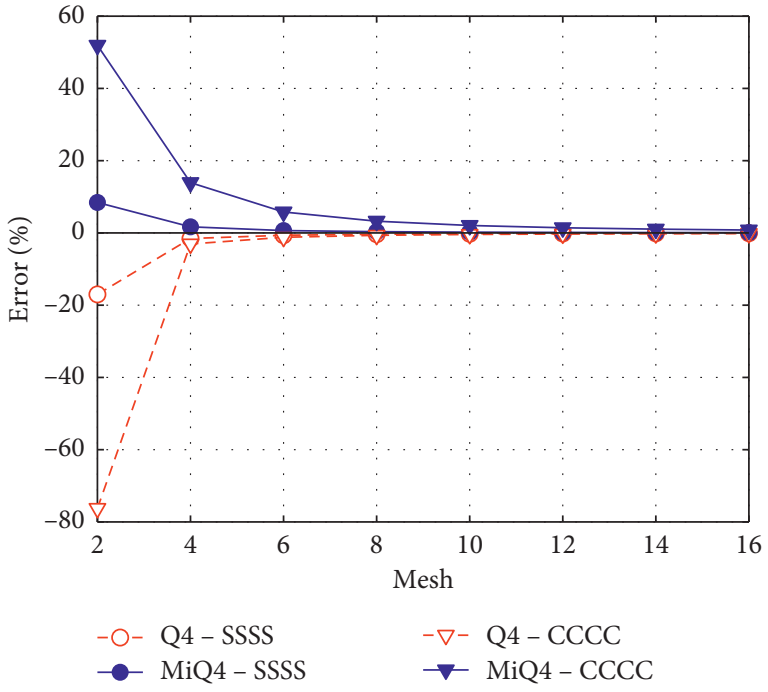

(a)

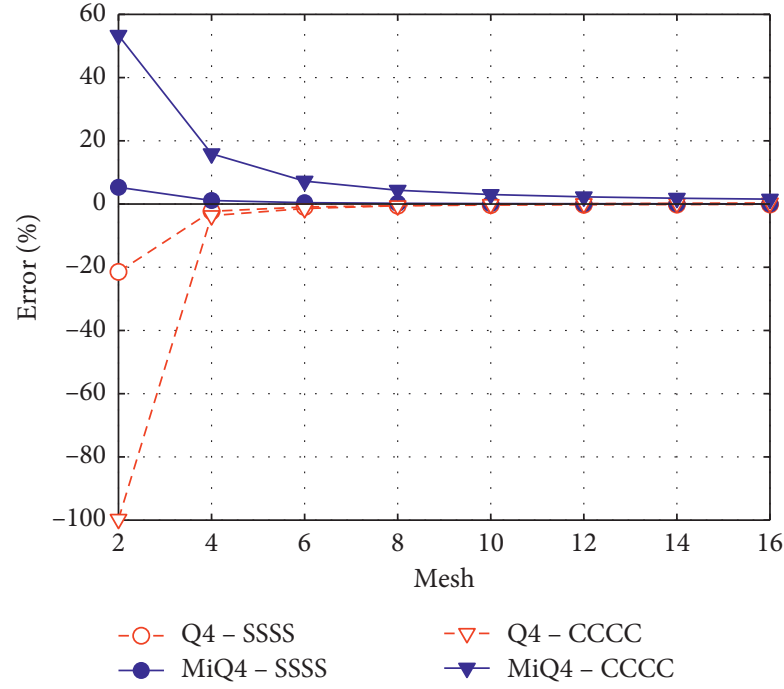

(b)

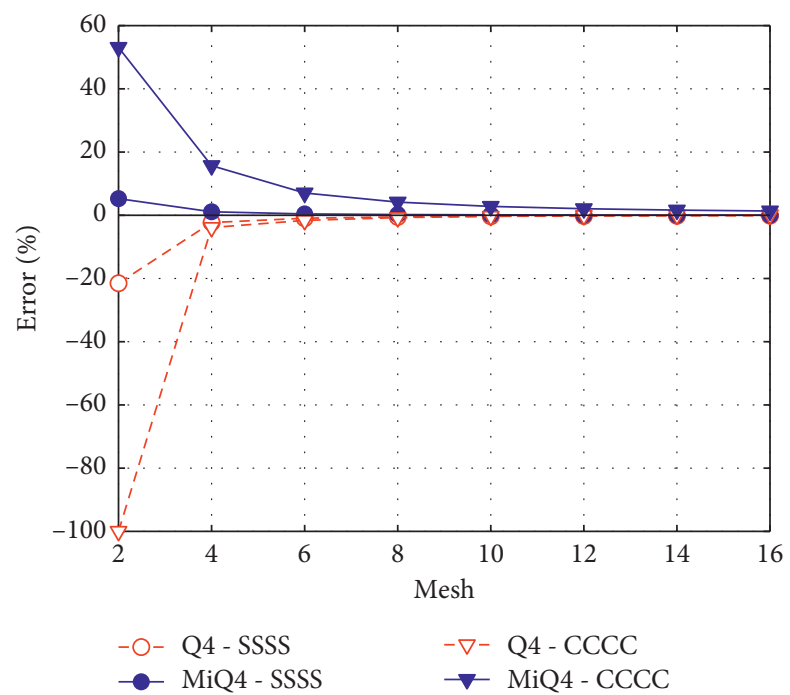

(c)

Figure 3: Comparison of central deflection $\left(w_{c} .100 \mathrm{D} / \mathrm{qa}^{4}\right)$ of the square plate: (a) $h / a=0.1$, (b) $h / a=0.01$, and (c) $h / a=0.001$.

correctly when the thickness-to-side ratio is smaller than $h / a=10^{-8}$.

\subsection{Distorted Study}

5.2.1. Morley's Acute Skew Plate. Continuously, we consider a skew plate (see Figure 4) subjected to a uniform load which is carried out by Morley [63]. The skew angle of the plate is $\beta=30^{\circ}$ and the BCs of the plate are soft simply supported $(w=0)$ at all edges. The geometry and material properties of the plate are $a=100, E=10.92$, and $\nu=0.3$ and the side-tothickness ratio $a / h$ is 100 and 1000 .

The comparisons of the nondimensional central deflection $\left(w^{*}=w_{c} 10^{3} \mathrm{D} / \mathrm{qa}^{4}\right)$ of the acute skew plates using the proposed element MiQ4, the numerical results using DKMQ [61], and those of MITC4 [61] element are presented in Tables 5 and 6 and Figure 5, in which the error of the numerical results is calculated in comparison with the solutions of Morley [63]. The comparison shows that the results of MiQ4 are in good agreement with the reference solution of Morley [63]. According to Tables 5 and 6, it can be seen that the convergence of the proposed element MiQ4 element for both thin and very thin plate is similar. The convergence of the proposed element MiQ4 is better than that of DKMQ and equable with MITC4 for thin plates $(a / h=100)$. For the case of very thin plates $(a / h=1000)$, the convergence of the present element MiQ4 is better than that of both DKMQ and MITC4 elements. Hence, the MiQ4 element can be applied to analyze both thin and very thin plates.

5.2.2. Square Plates with Severe Mesh Distortion. To exhibit the advantage of the MiQ4 element in comparison with the classical Q4 elements, a fully simply supported square plate is investigated with four types of distorted mesh. The 
TABle 4: Nondimensional central deflection $\left(w_{c} .100 \mathrm{D} / \mathrm{qa}^{4}\right)$ of the square plate with different values of $h / a$.

\begin{tabular}{lccccccc}
\hline BCs & $h / a$ & $10^{-3}$ & $10^{-4}$ & $10^{-6}$ & $10^{-8}$ & $10^{-10}$ & $10^{-30}$ \\
\hline \multirow{4}{*}{ SSSS } & Q4 & 0.405711 & 0.405709 & 0.405701 & 0.332142 & $0.1379 .10^{2}$ & $0.2206 .10^{-41}$ \\
& MiQ4 & 0.406453 & 0.406451 & 0.406451 & 0.406451 & 0.406451 & 0.406451 \\
& Cen et al. [59] & 0.40624 & 0.40624 & 0.40624 & 0.40624 & 0.40624 & 0.40624 \\
& Analytical [59] & 0.4062 & 0.4062 & 0.4062 & 0.4062 & 0.4062 & 0.4062 \\
\hline \multirow{4}{*}{ CCCC } & Q4 & 0.126163 & 0.126161 & 0.126159 & 0.117594 & 0.440847 & $0.9374 .10^{-39}$ \\
& MiQ4 & 0.127701 & 0.127698 & 0.127698 & 0.127698 & 0.127698 & 0.127698 \\
& Cen et al. [59] & 0.12653 & 0.12653 & 0.12653 & 0.12653 & 0.12653 & 0.12653 \\
& Analytical [59] & 0.1265 & 0.1265 & 0.1265 & 0.1265 & 0.1265 & 0.1265 \\
\hline
\end{tabular}

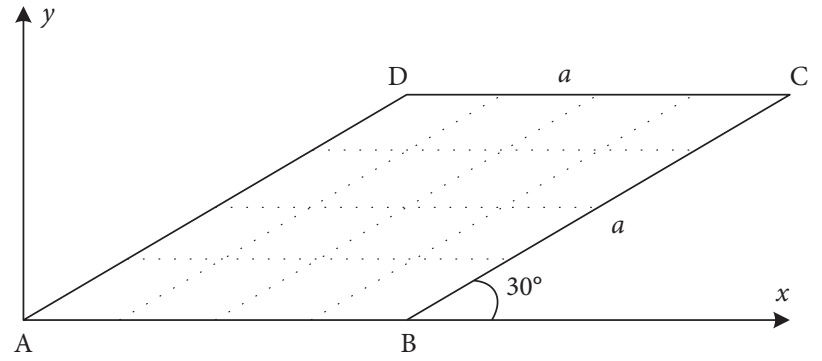

FIgURE 4: The model of Morley's acute plate.

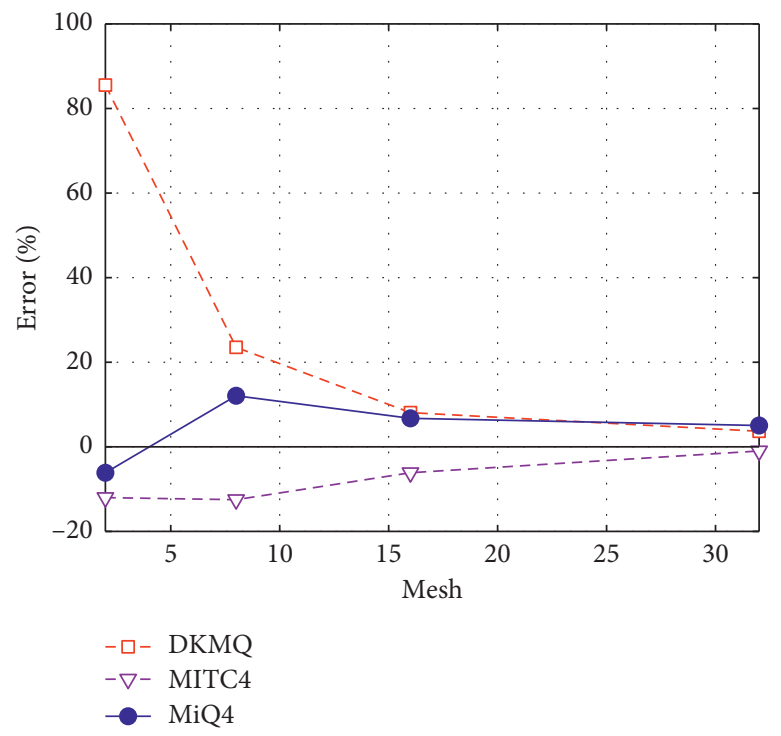

(a)

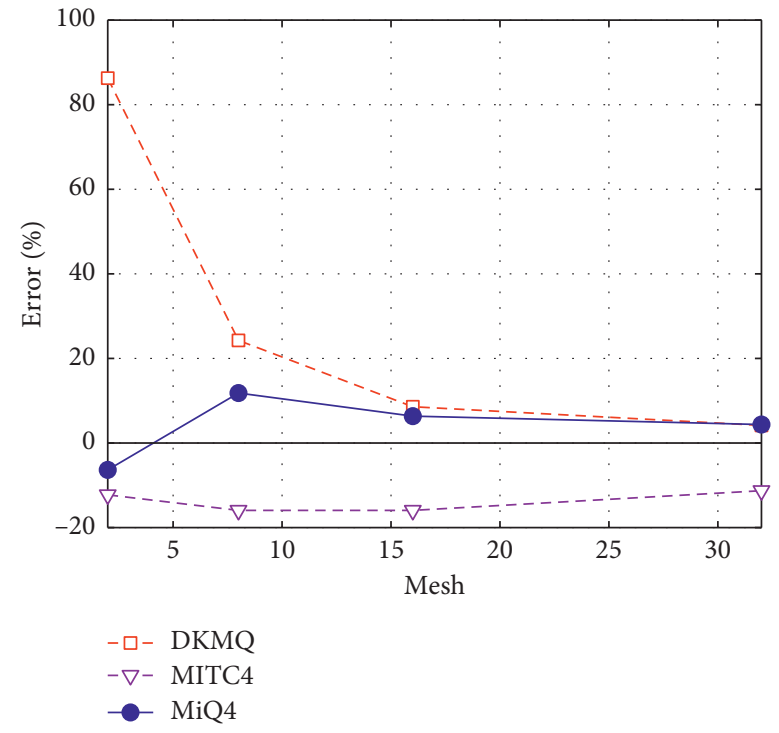

(b)

Figure 5: Comparison of the central deflection of Morley's acute plate subjected to uniform load: (a) $a / h=100$ and (b) $a / h=1000$.

TABLE 5: Central deflection of Morley's acute plate subjected to uniform load with $a / h=100$.

\begin{tabular}{lcccccc}
\hline Mesh & DKMQ [61] & Error (\%) & MITC4 [61] & Error (\%) & MiQ4 & Error (\%) \\
\hline $2 \times 2$ & 0.7570 & 85.54 & 0.3590 & -12.01 & 0.3830 \\
$8 \times 8$ & 0.5040 & 23.53 & 0.3570 & -12.50 & 0.4573 \\
$16 \times 16$ & 0.4410 & 8.09 & 0.3830 & -6.13 & 0.4355 \\
$32 \times 32$ & 0.4230 & 3.68 & 0.4040 & -0.98 & 0.4286 \\
Morley [63] & & & & 0.408 & & \\
\hline
\end{tabular}


TABLE 6: Central deflection of Morley's acute plate subjected to uniform load with $a / h=1000$.

\begin{tabular}{lcccccc}
\hline Mesh & DKMQ [61] & Error (\%) & MITC4 [61] & Error (\%) & MiQ4 & Error (\%) \\
\hline $2 \times 2$ & 0.7600 & 86.27 & 0.3580 & -12.25 & 0.3821 & -6.36 \\
$8 \times 8$ & 0.5070 & 24.26 & 0.3430 & -15.93 & 0.4562 \\
$16 \times 16$ & 0.4430 & 8.58 & 0.3430 & -15.93 & 0.4339 \\
$32 \times 32$ & 0.4250 & 4.17 & 0.3620 & -11.27 & 0.4258 \\
Morley [63] & & & & 0.408 & & \\
\hline
\end{tabular}

dimension and material properties of the plate are $a=10$, $E=10.92$, and $v=0.3$ and the thickness-to-side ratio $h / a$ is $0.1,0.01$, and 0.001 . Four types of distorted mesh are displaced in Figure 6 for the case of 64 elements. The nondimensional central deflection of the plates using MiQ4 and Q4 and the reference solutions [60] are presented in Tables 7-10 and Figure 7, where the error is calculated in comparison with the reference solutions [60] and those of Cen [59]. In comparison with Q4, MiQ4 is survival for all cases of distorted mesh, especially in the case of very thin plates, while the classical element Q4 cannot work accurately. This is a significant benefit of MiQ4 elements because the process of meshing always takes much time and computation cost in finite element analysis. Besides, the proposed element MiQ4 is suitable for the analysis of plates with arbitrary and complex geometries.

\subsection{Validation Study}

5.3.1. Bending of Isotropic Homogeneous Plates. In this subsection, the static bending of an isotropic homogeneous plate under uniform load is considered to prove the validity of the new plate element MiQ4. The geometrical and material properties of the quadratic plate are $a=b=1, E=1$, $q=1$, and $v=0.3$, while the thickness of the plate is considered by three values of $h=0.01, h=0.03$, and $h=0.1$. The comparison of the numerical results of the plates using the proposed element MiQ4 with other published results is presented in Table 11. It is obvious that the numerical results of the plates using the proposed element agree very well with those of CPT [26], the results of Nguyen et al. [18] using a refined simple FSDT, the solutions of Zenkour [26] using GSDT, and the 3D exact solutions [26]. In comparison with $3 \mathrm{D}$ solutions, the presented numerical results are minor difference.

5.3.2. Bending of Isotropic FGM Plates. Continuously, an FGM square plate of $\mathrm{Al} / \mathrm{Al}_{2} \mathrm{O}_{3}$ subjected to uniform load and sinusoidal load is considered to exhibit the accuracy of the proposed element MiQ4. Young's modulus is $E_{m}=70 \mathrm{GPa}$ for $\mathrm{Al}$ and $E_{c}=380 \mathrm{GPa}$ for $\mathrm{Al}_{2} \mathrm{O}_{3}$, while Poisson's ratios $v$ are constant and equal 0.3 , and the side-to-thickness ratio is $a / h=10$. The material properties of the plate vary through the thickness direction by the power-law function. The comparison of nondimensional displacement and stresses using MiQ4 and those of Zenkour [26] is shown in Table 12. The comparison shows that the numerical results of the plate using MiQ4 are identical to those of Zenkour [26] for both uniform and sinusoidal loads. The maximum error of the deflection is $1.35 \%$, while the maximum error of the stresses is $1.83 \%$. Therefore, it can conclude that the proposed element is compatible to analyze the FGM structure. The nondimensional quantities are obtained as the following formulae:

$$
\begin{gathered}
\bar{w}=\frac{10 E_{c} h^{3}}{q_{0} a^{4}} w\left(\frac{a}{2}, \frac{b}{2}\right), \\
\bar{\sigma}_{x}=\frac{h}{q_{0} a} \sigma_{x}\left(\frac{a}{2}, \frac{b}{2}, \frac{h}{2}\right), \\
\bar{\sigma}_{y}=\frac{h}{q_{0} a} \sigma_{y}\left(\frac{a}{2}, \frac{b}{2}, \frac{h}{3}\right), \\
\bar{\tau}_{x y}=\frac{h}{q_{0} a} \tau_{x y}\left(0,0,-\frac{h}{3}\right) .
\end{gathered}
$$

5.4. Parameter Study. The proposed plate element MiQ4 is now applied to examine the static bending behavior of variable thickness FGM plates subjected to uniform load. The ingredients of the FGM plates are $\mathrm{Al}$ as metal and $\mathrm{Al}_{2} \mathrm{O}_{3}$ as ceramic. Young's modulus is $E_{m}=70 \mathrm{GPa}$ for $\mathrm{Al}$ and $E_{c}=$ $380 \mathrm{GPa}$ for $\mathrm{Al}_{2} \mathrm{O}_{3}$, while Poisson's ratios $v$ are constant and equal to 0.3 , and the side-to-thickness ratio is $a / h_{0}=10$. The material properties of the plate vary through the thickness direction by the power-law function. The nondimensional quantities are computed as the following formulae:

$$
\begin{aligned}
w_{\text {max }}^{*} & =\frac{10 E_{c} h_{0}^{3}}{q_{0} a^{4}} w_{\max }, \\
\sigma_{x}^{*} & =\frac{h_{0}}{q_{0} a} \sigma_{x}\left(\frac{a}{2}, \frac{b}{2}, z\right), \\
\tau_{x y}^{*} & =\frac{h_{0}}{q_{0} a} \tau_{x y}(a, 0, z) .
\end{aligned}
$$

It is noticed that the proposed element, MiQ4, is of good accuracy and convergence with coarse meshes, but the plates are divided with a fine mesh because of its thickness variation. Accordingly, a regular fine mesh of $N \times N=32 \times 32$ is handled in the rest of this study.

5.4.1. The Influence of the Power-Law Index. Firstly, we study the effects of the power-law index $p$ on the deflection of the variable thickness FGM plates. A square FGM plate with the 


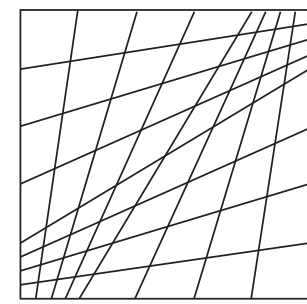

Type A

(a)

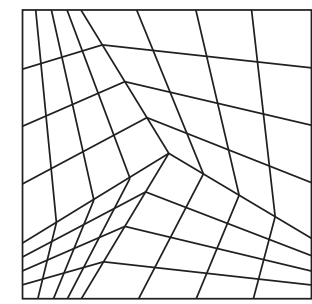

Type B

(b)

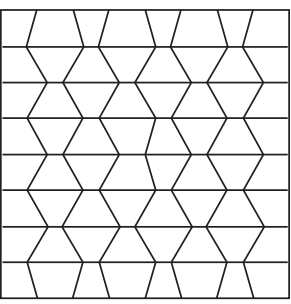

Type C

(c)

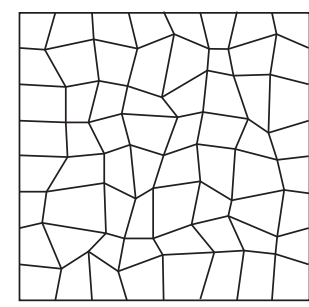

Type D

(d)

Figure 6: Four types of distorted mesh of square plates.

TABLE 7: Central deflection $\left(w_{c} \cdot 100 \mathrm{D} / \mathrm{qa}^{4}\right)$ of the square SSSS plates with $h / a=0.1$.

\begin{tabular}{lcccccrrr}
\hline \multirow{2}{*}{ Mesh } & \multicolumn{2}{c}{ Type A } & \multicolumn{2}{c}{ Type B } & \multicolumn{2}{c}{ Type C } & \multicolumn{2}{c}{ Type D } \\
& Q4 & MiQ4 & Q4 & MiQ4 & Q4 & MiQ4 & Q4 \\
\hline $4 \times 4$ & 0.391576 & 0.439143 & 0.378981 & 0.421275 & 0.400312 & 0.426277 & 0.355584 \\
$8 \times 8$ & 0.419534 & 0.429446 & 0.417878 & 0.425853 & 0.414445 & 0.423622 & 0.403264 \\
$16 \times 16$ & 0.425205 & 0.427616 & 0.425021 & 0.426912 & 0.423067 & 0.425691 & 0.417927 & 0.42214376 \\
$32 \times 32$ & 0.426749 & 0.427352 & 0.426715 & 0.427183 & 0.426074 & 0.426782 & 0.427397 & 0.427632 \\
{$[60]$} & & & \multicolumn{7}{c}{0.427284} \\
\hline
\end{tabular}

TABLE 8: Central deflection $\left(w_{c} \cdot 100 \mathrm{D} / \mathrm{qa}^{4}\right)$ of the square SSSS plates with $h / a=0.01$.

\begin{tabular}{lcccccrrr}
\hline \multirow{2}{*}{ Mesh } & \multicolumn{2}{c}{ Type A } & \multicolumn{2}{c}{ Type B } & \multicolumn{2}{c}{ Type C } & \multicolumn{2}{c}{ Type D } \\
& Q4 & MiQ4 & Q4 & MiQ4 & Q4 & MiQ4 & Q4 \\
\hline $4 \times 4$ & 0.239679 & 0.409780 & 0.227367 & 0.393247 & 0.116995 & 0.400856 & 0.088091 \\
$8 \times 8$ & 0.380737 & 0.407129 & 0.385881 & 0.403634 & 0.186903 & 0.395633 & 0.197400 & 0.363006 \\
$16 \times 16$ & 0.403429 & 0.406497 & 0.403369 & 0.405834 & 0.264044 & 0.394633 & 0.287950 & 0.388361 \\
$32 \times 32$ & 0.405812 & 0.406450 & 0.405804 & 0.406299 & 0.340033 & 0.396093 & 0.356015 & 0.395804 \\
{$[60]$} & & \multicolumn{3}{c}{0.406446} \\
\hline
\end{tabular}

TABLE 9: Central deflection $\left(w_{c} .100 \mathrm{D} / \mathrm{qa}^{4}\right)$ of the square plates with $h / a=0.001$.

\begin{tabular}{lcccccrrr}
\hline \multirow{2}{*}{ Mesh } & \multicolumn{2}{c}{ Type A } & \multicolumn{2}{c}{ Type B } & \multicolumn{2}{c}{ Type C } & \multicolumn{2}{c}{ Type D } \\
& Q4 & MiQ4 & Q4 & MiQ4 & Q4 & MiQ4 & Q4 \\
\hline $4 \times 4$ & 0.114621 & 0.409454 & 0.142155 & 0.392942 & 0.034504 & 0.400589 & 0.026915 \\
$8 \times 8$ & 0.323262 & 0.406892 & 0.363450 & 0.403385 & 0.094029 & 0.395205 & 0.101194 & 0.362634 \\
$16 \times 16$ & 0.391383 & 0.406280 & 0.398910 & 0.405607 & 0.141653 & 0.393440 & 0.177568 & 0.386775 \\
$32 \times 32$ & 0.404963 & 0.406239 & 0.405168 & 0.406084 & 0.172764 & 0.392416 & 0.216136 & 0.391490 \\
{$[60]$} & & & \multicolumn{7}{c}{0.406237} \\
\hline
\end{tabular}

TABLE 10: Central deflection $\left(w_{c} .100 \mathrm{D} / \mathrm{qa}^{4}\right)$ of the square SSSS plates with $h / a=10^{-6}$.

\begin{tabular}{|c|c|c|c|c|c|c|c|c|}
\hline \multirow{2}{*}{ Mesh } & \multicolumn{2}{|c|}{ Type A } & \multicolumn{2}{|c|}{ Type B } & \multicolumn{2}{|c|}{ Type C } & \multicolumn{2}{|c|}{ Type D } \\
\hline & Q4 & MiQ4 & Q4 & MiQ4 & Q4 & MiQ4 & Q4 & MiQ4 \\
\hline $4 \times 4$ & 0.118006 & 0.409451 & 0.110797 & 0.392939 & 0.024006 & 0.400587 & 0.020252 & 0.362630 \\
\hline $8 \times 8$ & 0.318335 & 0.406889 & 0.370621 & 0.403382 & 0.058159 & 0.395200 & 0.113783 & 0.380712 \\
\hline $16 \times 16$ & 0.000000 & 0.406278 & 0.723266 & 0.405605 & 0.132256 & 0.393427 & 0.121722 & 0.386758 \\
\hline $32 \times 32$ & 0.382467 & 0.406237 & 0.000000 & 0.406082 & 0.328034 & 0.392366 & 0.000000 & 0.391430 \\
\hline [59] & \multicolumn{8}{|c|}{0.40624} \\
\hline
\end{tabular}




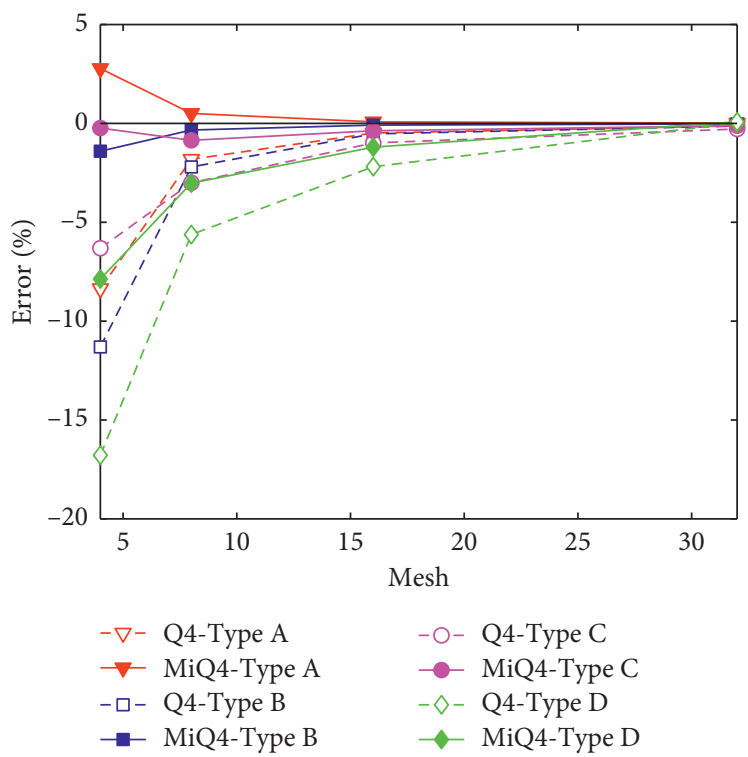

(a)

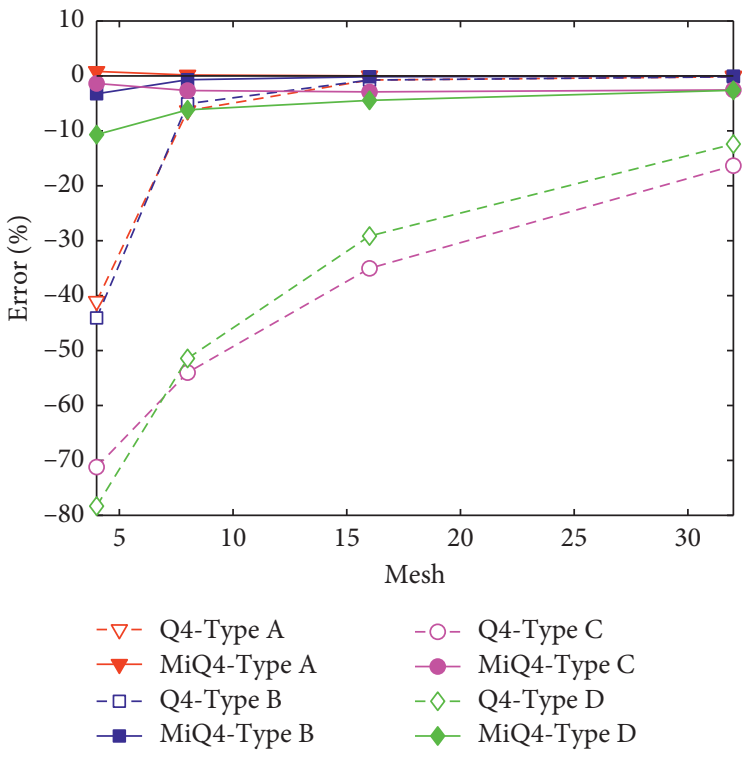

(b)

Figure 7: Continued. 


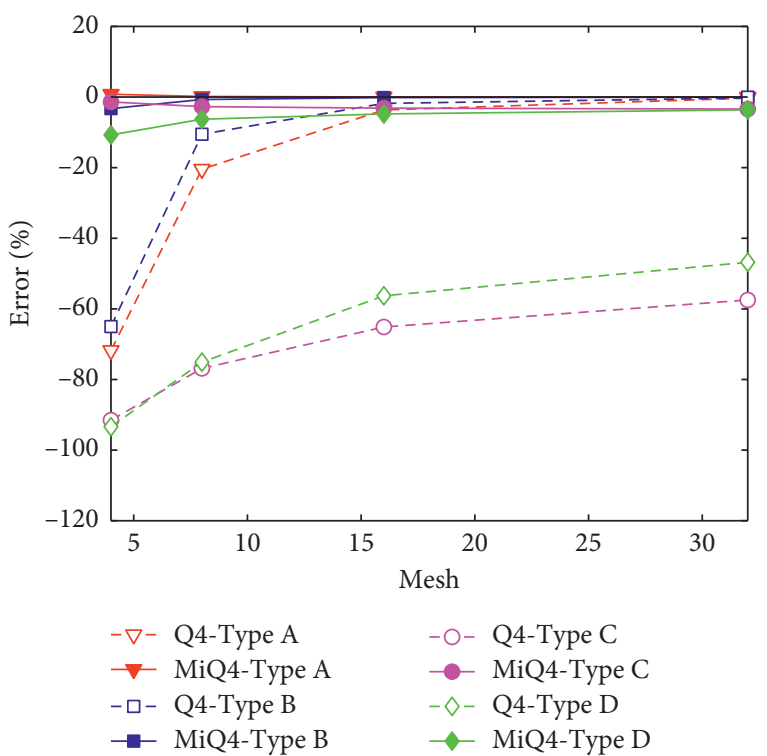

(c)

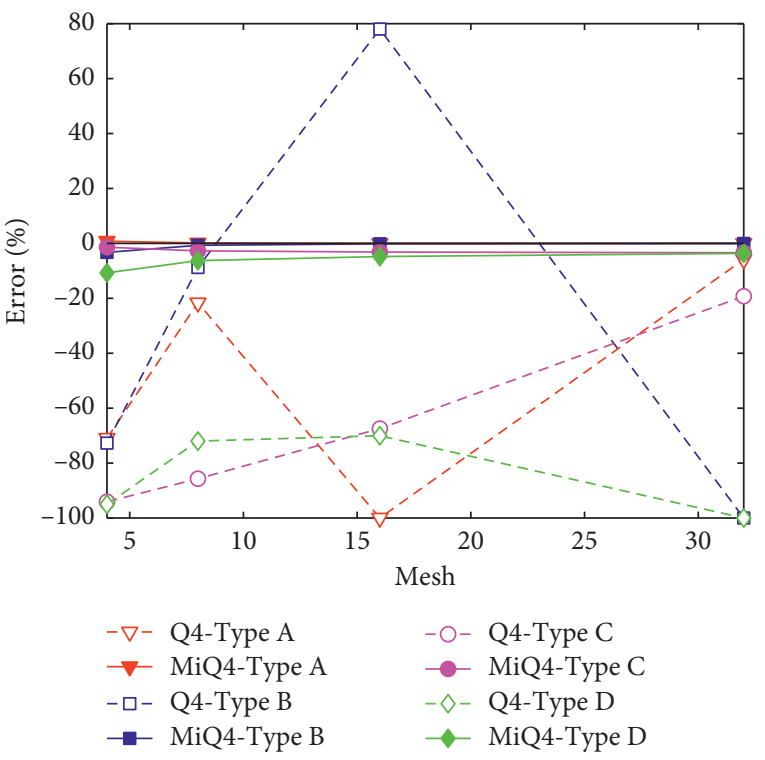

(d)

Figure 7: Comparison of central deflection of the square SSSS plates: (a) $h / a=0.1$, (b) $h / a=0.01$, (c) $h / a=0.001$, (d) $h / a=10^{-6}$.

TABLE 11: The central deflection of isotropic homogeneous plates under uniform load.

\begin{tabular}{lcccccc}
\hline$h$ & Classical [26] & RS-FSDT [18] & SSDT [26] & 3D [26] & Present & Different with 3D (\%) \\
\hline 0.01 & 44360.9 & 44385.41 & 44383.84 & 44384.7 & 44388.77 & -0.009 \\
0.03 & 1643.00 & 1651.169 & 1650.646 & 1650.94 & 1650.85 & -0.005 \\
0.1 & 44.3609 & 46.81271 & 46.65481 & 46.7443 & 46.6678 & -0.164 \\
\hline
\end{tabular}


TABLE 12: Nondimensional deflection and stresses of the square SSSS FGM plates.

\begin{tabular}{|c|c|c|c|c|c|c|c|c|c|}
\hline \multirow{2}{*}{$p$} & \multirow{2}{*}{ Method } & \multicolumn{4}{|c|}{ Uniform load } & \multicolumn{4}{|c|}{ Sinusoidal load } \\
\hline & & $\bar{w}$ & $\bar{\sigma}_{x}$ & $\bar{\sigma}_{y}$ & $\bar{\tau}_{x y}$ & $\bar{w}$ & $\bar{\sigma}_{x}$ & $\bar{\sigma}_{y}$ & $\bar{\tau}_{x y}$ \\
\hline \multirow{3}{*}{ Ceramic } & Zenkour [26] & 0.4665 & 2.8932 & 1.9103 & 1.2850 & 0.2960 & 1.9955 & 1.3121 & 0.7065 \\
\hline & Present & 0.4667 & 2.8696 & 1.9131 & 1.2871 & 0.2960 & 1.9697 & 1.3131 & 0.7071 \\
\hline & Error (\%) & 0.04 & -0.82 & 0.15 & 0.16 & -0.00 & -1.29 & 0.08 & 0.08 \\
\hline \multirow{3}{*}{1} & Zenkour [26] & 0.9287 & 4.4745 & 2.1692 & 1.1143 & 0.5889 & 3.0870 & 1.4894 & 0.6110 \\
\hline & Present & 0.9289 & 4.4351 & 2.1738 & 1.1120 & 0.5887 & 3.0442 & 1.4921 & 0.6107 \\
\hline & Error (\%) & 0.02 & -0.88 & 0.21 & -0.21 & -0.03 & -1.39 & 0.18 & -0.05 \\
\hline \multirow{3}{*}{2} & Zenkour [26] & 1.1940 & 5.2296 & 2.0338 & 0.9907 & 0.7573 & 3.6094 & 1.3954 & 0.5441 \\
\hline & Present & 1.1910 & 5.1786 & 2.0414 & 0.9911 & 0.7548 & 3.5546 & 1.4012 & 0.5443 \\
\hline & Error (\%) & -0.25 & -0.97 & 0.37 & 0.04 & -0.32 & -1.52 & 0.41 & 0.04 \\
\hline \multirow{3}{*}{3} & Zenkour [26] & 1.3200 & 5.6108 & 1.8593 & 1.0047 & 0.8377 & 3.8742 & 1.2748 & 0.5525 \\
\hline & Present & 1.3124 & 5.5504 & 1.8693 & 1.0071 & 0.8320 & 3.8098 & 1.2831 & 0.5531 \\
\hline & Error (\%) & -0.57 & -1.08 & 0.54 & 0.24 & -0.68 & -1.66 & 0.65 & 0.11 \\
\hline \multirow{3}{*}{4} & Zenkour [26] & 1.3890 & 5.8915 & 1.7197 & 1.0298 & 0.8819 & 4.0693 & 1.1783 & 0.5667 \\
\hline & Present & 1.3771 & 5.8240 & 1.7314 & 1.0336 & 0.8732 & 3.9975 & 1.1884 & 0.5676 \\
\hline & Error (\%) & -0.86 & -1.15 & 0.68 & 0.37 & -0.99 & -1.76 & 0.86 & 0.17 \\
\hline \multirow{3}{*}{5} & Zenkour [26] & 1.4356 & 6.1504 & 1.6104 & 1.0451 & 0.9118 & 4.2488 & 1.1029 & 0.5755 \\
\hline & Present & 1.4206 & 6.0778 & 1.6230 & 1.0498 & 0.9010 & 4.1717 & 1.1140 & 0.5766 \\
\hline & Error (\%) & -1.04 & -1.18 & 0.78 & 0.45 & -1.18 & -1.81 & 1.00 & 0.18 \\
\hline \multirow{3}{*}{6} & Zenkour [26] & 1.4727 & 6.4043 & 1.5214 & 1.0536 & 0.9356 & 4.4244 & 1.0417 & 0.5803 \\
\hline & Present & 1.4557 & 6.3281 & 1.5343 & 1.0589 & 0.9234 & 4.3436 & 1.0531 & 0.5816 \\
\hline & Error (\%) & -1.16 & -1.19 & 0.85 & 0.51 & -1.30 & -1.83 & 1.10 & 0.22 \\
\hline \multirow{3}{*}{7} & Zenkour [26] & 1.5049 & 6.6547 & 1.4467 & 1.0589 & 0.9562 & 4.5971 & 0.9903 & 0.5834 \\
\hline & Present & 1.4869 & 6.5761 & 1.4595 & 1.0646 & 0.9433 & 4.5138 & 1.0018 & 0.5847 \\
\hline & Error (\%) & -1.20 & -1.18 & 0.88 & 0.54 & -1.35 & -1.81 & 1.16 & 0.22 \\
\hline \multirow{3}{*}{8} & Zenkour [26] & 1.5343 & 6.8999 & 1.3829 & 1.0628 & 0.9750 & 4.7661 & 0.9466 & 0.5856 \\
\hline & Present & 1.5159 & 6.8197 & 1.3953 & 1.0687 & 0.9619 & 4.6810 & 0.9577 & 0.5870 \\
\hline & Error (\%) & -1.20 & -1.16 & 0.90 & 0.56 & -1.35 & -1.79 & 1.18 & 0.23 \\
\hline \multirow{3}{*}{9} & Zenkour [26] & 1.5617 & 7.1383 & 1.3283 & 1.0662 & 0.9925 & 4.9303 & 0.9092 & 0.5875 \\
\hline & Present & 1.5435 & 7.0570 & 1.3404 & 1.0723 & 0.9794 & 4.8438 & 0.9200 & 0.5889 \\
\hline & Error (\%) & -1.17 & -1.14 & 0.91 & 0.57 & -1.32 & -1.75 & 1.19 & 0.24 \\
\hline \multirow{3}{*}{10} & Zenkour [26] & 1.5876 & 7.3689 & 1.2820 & 1.0694 & 1.0089 & 5.0890 & 0.8775 & 0.5894 \\
\hline & Present & 1.5698 & 7.2867 & 1.2935 & 1.0757 & 0.9962 & 5.0015 & 0.8879 & 0.5908 \\
\hline & Error (\%) & -1.12 & -1.12 & 0.90 & 0.59 & -1.26 & -1.72 & 1.18 & 0.24 \\
\hline \multirow{3}{*}{ Metal } & Zenkour [26] & 2.5327 & 2.8932 & 1.9103 & 1.2850 & 1.6070 & 1.9955 & 1.3121 & 0.7065 \\
\hline & Present & 2.5334 & 2.8696 & 1.9131 & 1.2871 & 1.6066 & 1.9697 & 1.3131 & 0.7071 \\
\hline & Error (\%) & 0.03 & -0.82 & 0.15 & 0.16 & -0.02 & -1.29 & 0.08 & 0.08 \\
\hline
\end{tabular}

TABLE 13: The maximum nondimensional deflection of the FGM plates as a function of $p$.

\begin{tabular}{|c|c|c|c|c|c|c|c|}
\hline \multirow{2}{*}{$\mathrm{BCs}$} & \multirow{2}{*}{ Thickness variations } & \multicolumn{6}{|c|}{$p$} \\
\hline & & Ceramic & 0.5 & 1 & 5 & 10 & Metal \\
\hline \multirow{4}{*}{ CCCC } & U plate & 0.1646 & 0.2496 & 0.3221 & 0.5023 & 0.5627 & 0.8935 \\
\hline & L plate & 0.4010 & 0.6124 & 0.7927 & 1.2211 & 1.3567 & 2.1762 \\
\hline & $\mathrm{P}$ plate & 0.2894 & 0.4409 & 0.5700 & 0.8815 & 0.9822 & 1.5707 \\
\hline & S plate & 0.5501 & 0.8416 & 1.0901 & 1.6742 & 1.8563 & 2.9854 \\
\hline \multirow{4}{*}{ SCSC } & U plate & 0.2414 & 0.3672 & 0.4744 & 0.7363 & 0.8222 & 1.3104 \\
\hline & L plate & 0.5912 & 0.9044 & 1.1716 & 1.7997 & 1.9955 & 3.2085 \\
\hline & $\mathrm{P}$ plate & 0.4432 & 0.6769 & 0.8761 & 1.3490 & 1.4987 & 2.4054 \\
\hline & S plate & 0.7792 & 1.1933 & 1.5466 & 2.3707 & 2.6252 & 4.2286 \\
\hline \multirow{4}{*}{ SSSS } & U plate & 0.4667 & 0.7160 & 0.9289 & 1.4206 & 1.5698 & 2.5334 \\
\hline & L plate & 1.1636 & 1.7895 & 2.3240 & 3.5398 & 3.9007 & 6.3151 \\
\hline & $\mathrm{P}$ plate & 0.8203 & 1.2604 & 1.6361 & 2.4953 & 2.7526 & 4.4518 \\
\hline & S plate & 1.6256 & 2.5012 & 3.2487 & 4.9430 & 5.4438 & 8.8222 \\
\hline \multirow{4}{*}{ CFCF } & U plate & 0.3631 & 0.5529 & 0.7147 & 1.1068 & 1.2340 & 1.9708 \\
\hline & L plate & 1.6408 & 2.5181 & 3.2667 & 4.9911 & 5.5131 & 8.9048 \\
\hline & P plate & 1.2060 & 1.8494 & 2.3983 & 3.6692 & 4.0570 & 6.5452 \\
\hline & $S$ plate & 2.2183 & 3.4062 & 4.4195 & 6.7455 & 7.4466 & 12.0390 \\
\hline
\end{tabular}


TABLE 14: The maximum nondimensional deflection of the FGM plates as a function of $a / h_{0}$.

\begin{tabular}{|c|c|c|c|c|c|c|c|}
\hline \multirow{2}{*}{ BCs } & \multirow{2}{*}{ Thickness variations } & \multicolumn{6}{|c|}{$a / h_{0}$} \\
\hline & & 5 & 10 & 20 & 30 & 40 & 50 \\
\hline \multirow{4}{*}{ СССС } & U plate & 0.5745 & 0.4134 & 0.3707 & 0.3625 & 0.3596 & 0.3582 \\
\hline & L plate & 1.2445 & 1.0165 & 0.9565 & 0.9451 & 0.9410 & 0.9392 \\
\hline & P plate & 0.9339 & 0.7311 & 0.6775 & 0.6674 & 0.6638 & 0.6621 \\
\hline & S plate & 1.6532 & 1.3976 & 1.3304 & 1.3176 & 1.3131 & 1.3110 \\
\hline \multirow{4}{*}{ SCSC } & U plate & 0.8054 & 0.6087 & 0.5564 & 0.5465 & 0.5430 & 0.5414 \\
\hline & L plate & 1.7806 & 1.5023 & 1.4296 & 1.4159 & 1.4110 & 1.4088 \\
\hline & P plate & 1.3691 & 1.1233 & 1.0589 & 1.0467 & 1.0424 & 1.0404 \\
\hline & S plate & 2.2957 & 1.9826 & 1.9009 & 1.8855 & 1.8801 & 1.8776 \\
\hline \multirow{4}{*}{ SSSS } & U plate & 1.3424 & 1.1910 & 1.1531 & 1.1461 & 1.1437 & 1.1425 \\
\hline & L plate & 3.1909 & 2.9790 & 2.9259 & 2.9160 & 2.9126 & 2.9110 \\
\hline & P plate & 2.2864 & 2.0972 & 2.0498 & 2.0410 & 2.0379 & 2.0365 \\
\hline & S plate & 4.4033 & 4.1635 & 4.1031 & 4.0919 & 4.0880 & 4.0862 \\
\hline \multirow{4}{*}{ CFCF } & U plate & 1.1883 & 0.9169 & 0.8451 & 0.8307 & 0.8253 & 0.8227 \\
\hline & L plate & 4.6506 & 4.1874 & 4.0595 & 4.0331 & 4.0232 & 4.0184 \\
\hline & P plate & 3.4750 & 3.0744 & 2.9666 & 2.9449 & 2.9370 & 2.9331 \\
\hline & $S$ plate & 6.2105 & 5.6642 & 5.5084 & 5.4751 & 5.4624 & 5.4562 \\
\hline
\end{tabular}

\begin{tabular}{llll}
\hline Thickness & 3D views & $x z$ views & $y z$ views \\
variations & &
\end{tabular}

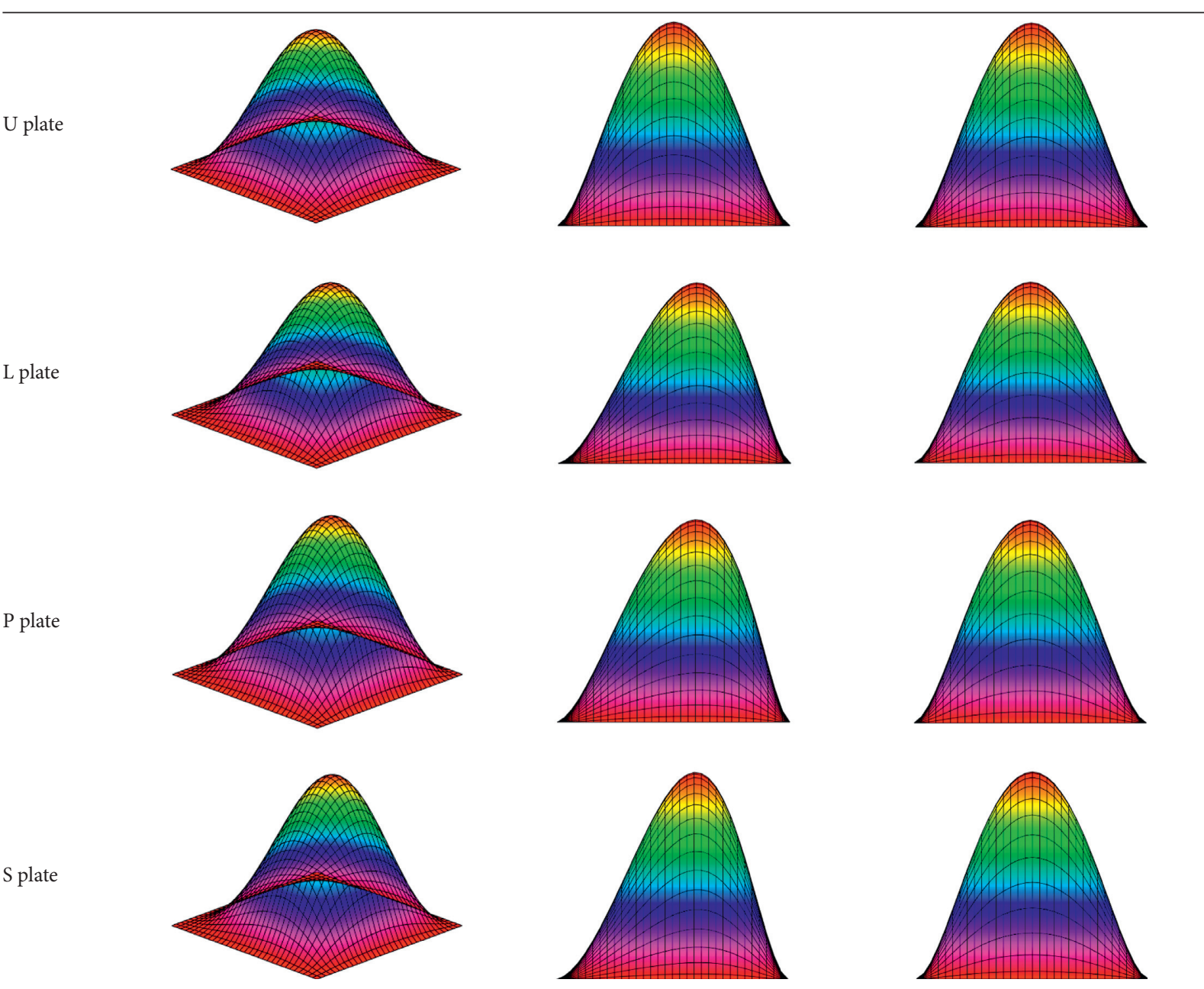

FIGURE 8: Deflected shapes of the CCCC FGM plates depend on the thickness variations. 


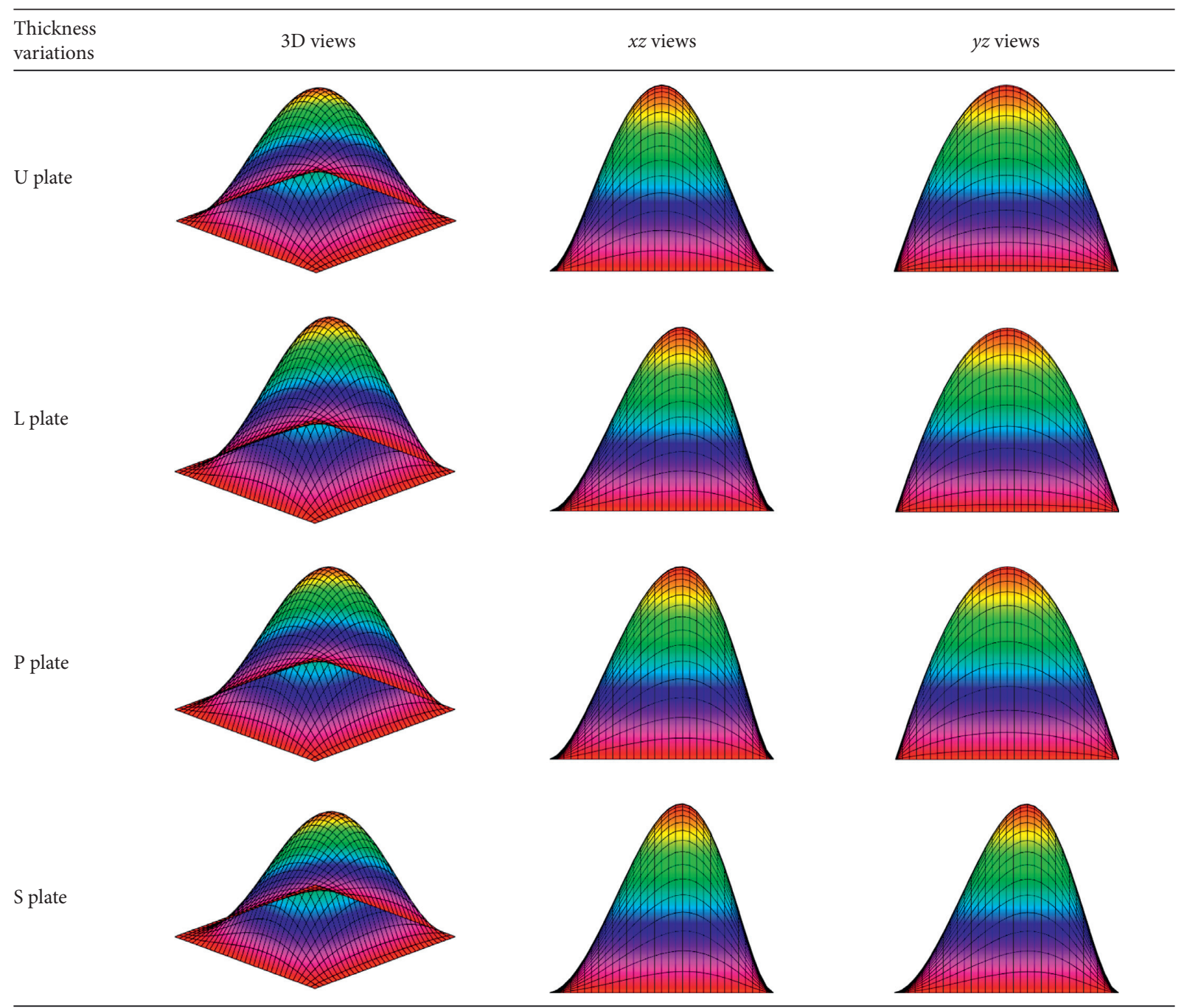

FIGURE 9: Deflected shapes of the SCSC FGM plates depend on the thickness variations.

side-to-thickness ratio of $a / h_{0}=10$ and the varied parameter $\zeta=0.5$ is considered. The maximum nondimensional deflections of the plate for four cases of the BCs including fully clamped plates (CCCC), two opposite sides are simply supported and two other sides are clamped plates (SCSC), fully simple supported plates (SSSS), and two opposite sides are clamped and two other sides are free plates (CFCF) with different values of the power-law index are presented in Table 13. According to Table 13, it can be seen clearly that the increasing power-law index leads to increasing the maximum deflection of the plates for all cases of BCs and four types of thickness variation. It is due to the fact that when the powerlaw index increases, the plate becomes a metal-rich plate. In a comparison of the deflection between four cases of thickness variation of the plates, it can be concluded that the maximum deflection of the $S$ plate is greatest, while the maximum deflection of the $\mathrm{U}$ plate is smallest.

5.4.2. The Eeffects of the Side-to-Thickness Ratio. Secondly, the effects of the side-to-thickness ratio $a / h_{0}$ on the deflection of the variable thickness FGM plates are investigated in this subsection. In this investigation, a square FGM plate with $p=2$ and $\zeta=0.5$ is considered. The maximum nondimensional deflections of the plate for four cases of the BCs and thickness variations with different values of the side-to-thickness ratio are shown in Table 14. It seems that the change of the side-to-thickness ratio has strong effects on the deflection of the plate. When the sideto-thickness ratio increases, the maximum nondimensional deflection of the plate decreases. In addition, the BCs affect strongly the deflection of the plates. The maximum deflection of the CCCC plate is smallest, while the maximum deflection of the CFCF plate is greatest.

5.4.3. The Influence of the Thickness Variation and BCs. Continuously, the effects of the thickness variation and BCs on the deflection and stresses of the variable thickness FGM plate are studied in this subsection. A square FGM plate with the power-law index of $p=1$, the varying parameter of $\zeta=0.5$, and the side-to-thickness ratio of $a / h_{0}=10$ is examined. The dependents of the deflected shapes on the BCs and the type of thickness variation are exhibited in 


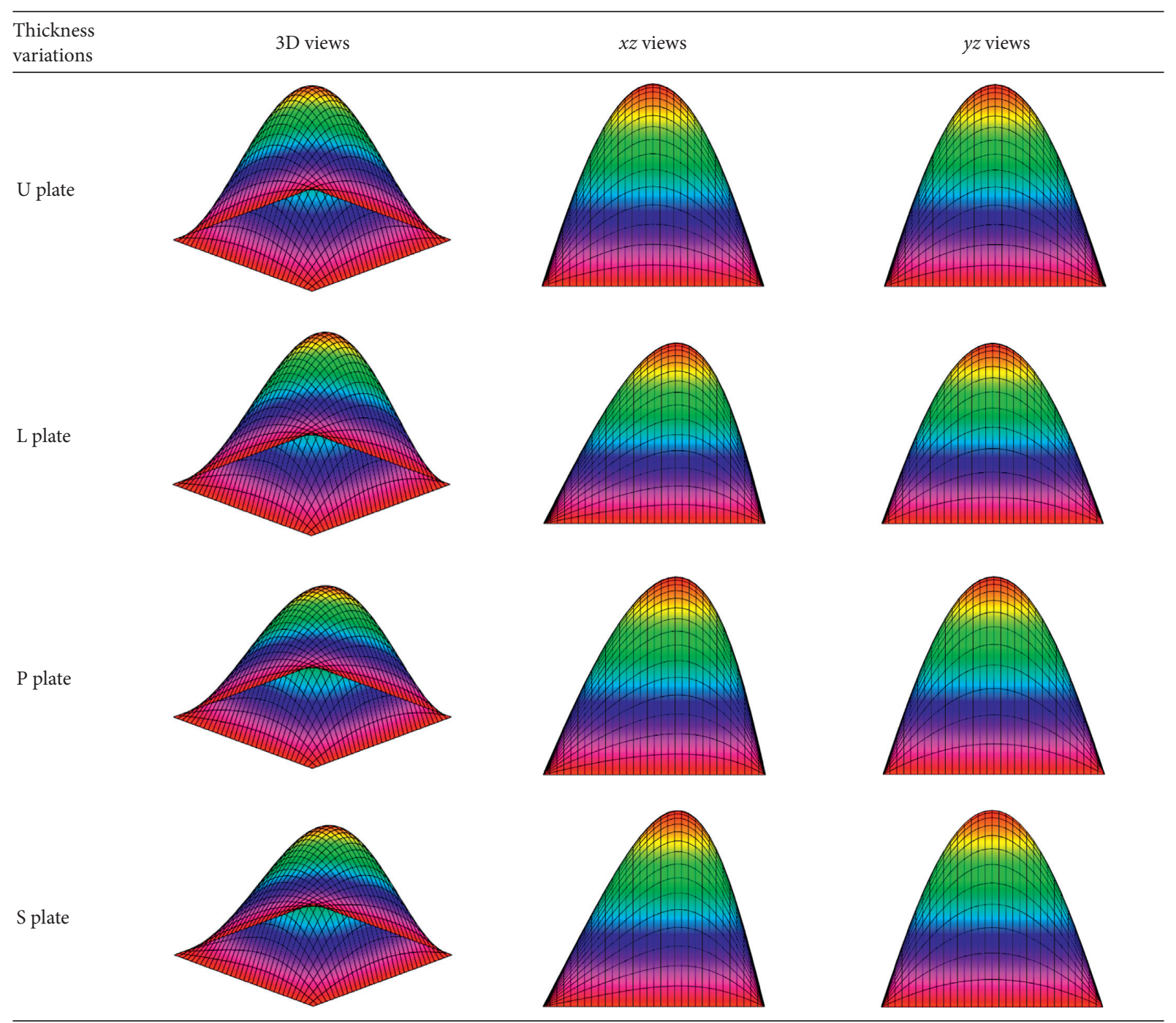

FIGURE 10: Deflected shapes of the SSSS FGM plates depend on the thickness variations.

Figures 8-11. The change of the BCs leads to the change of the deflected shape of the plates. Besides, the strong effects of the thickness variation on the deflection of the plates are that the maximum deflection of the varied plate will shift to the thin side of the plate except for $U$ plates. The deflected shapes are symmetrical in the case of $U$ plates, while they are asymmetrical in other cases. In the case of CFCF plates, the maximum deflection appears at the midpoint of the thin edge of the plates.

Additionally, we investigate the dependence of the stresses of the variable thickness FGM plate on the type of thickness variation. The nondimensional normal stress $\sigma_{x}^{*}$ and in-plane shear stress $\tau_{x y}^{*}$ of the plate for four cases of thickness variation are demonstrated in Figure 12 for the case of square SSSS FGM plate and $p=1, \zeta=0.5$. The stresses are strongly affected by the variation of the thickness. The maximum tensile normal stress and in-plane shear stress occur at the ceramic-rich surface of the plates.
Furthermore, the maximum stresses of the $\mathrm{S}$ plates are greatest in comparison to the other cases, so it can lead to the stress concentration in the variable thickness structures. The stress distribution also depends on the law of thickness variation. The stresses at the middle FGM plate does not equal zero as in isotropic homogeneous; it moves toward the ceramic-rich surface of the FGM plates; especially, in the case of $P$ plates, the normal stress equals zero at two points of the vertical fiber; it differs with other cases.

Finally, the effects of the varied parameter $\zeta$ on the maximum deflection of the plates are studied. A square FGM plate with $p=1$ and $a / h_{0}=10$ is considered. The varied parameter varies in the range of $0 \div 0.5$. It can see from Figure 13 that when the varied parameter increases, the maximum deflection of the plates increases rapidly for all cases of thickness variation except for $U$ plate. The speed of the increase depends on the type of the thickness variation in the order of $\mathrm{P}$ plate, L plate, and S plate. It also seems that the 


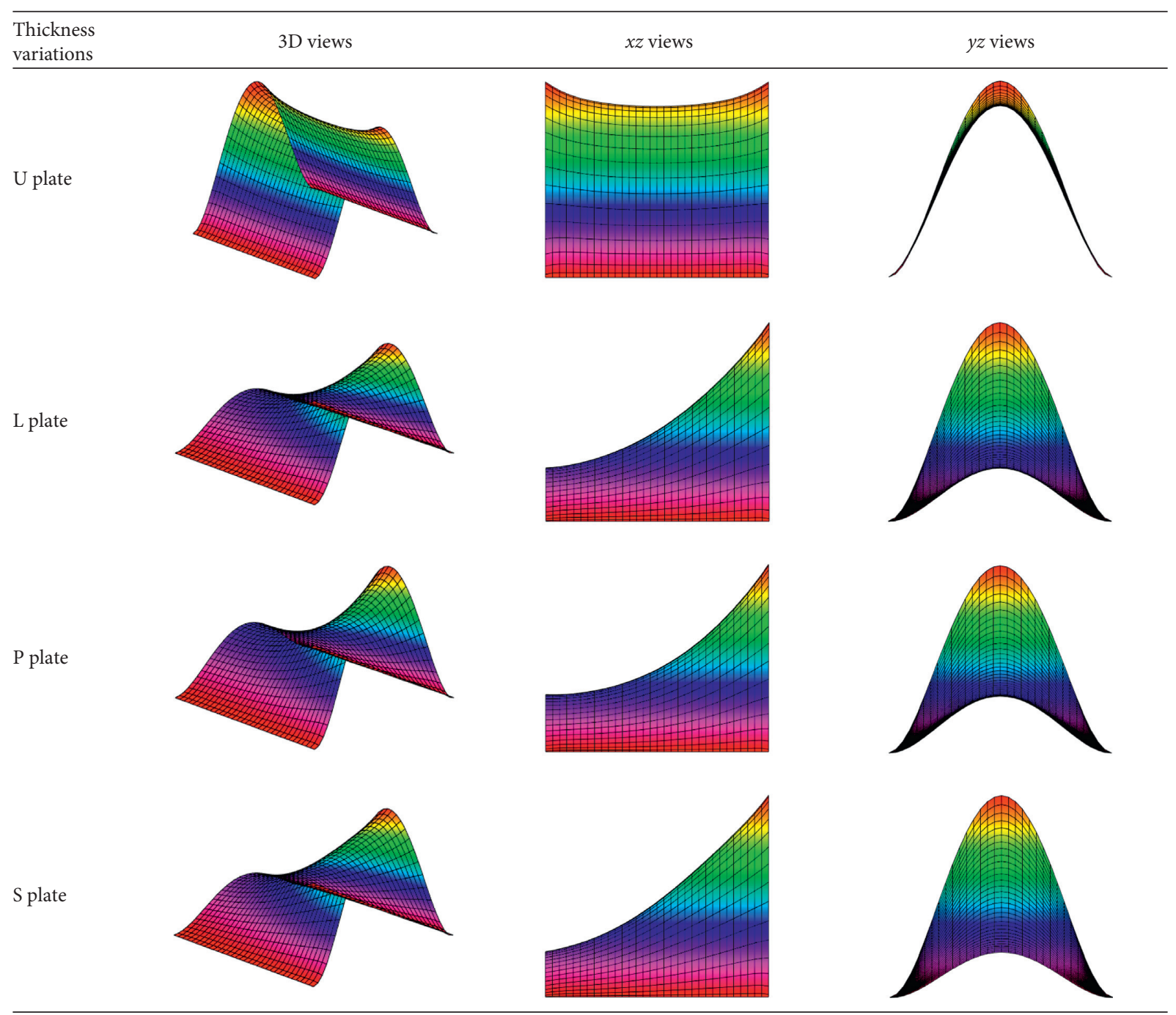

FIGURE 11: Deflected shapes of the CFCF FGM plates depend on the thickness variations.

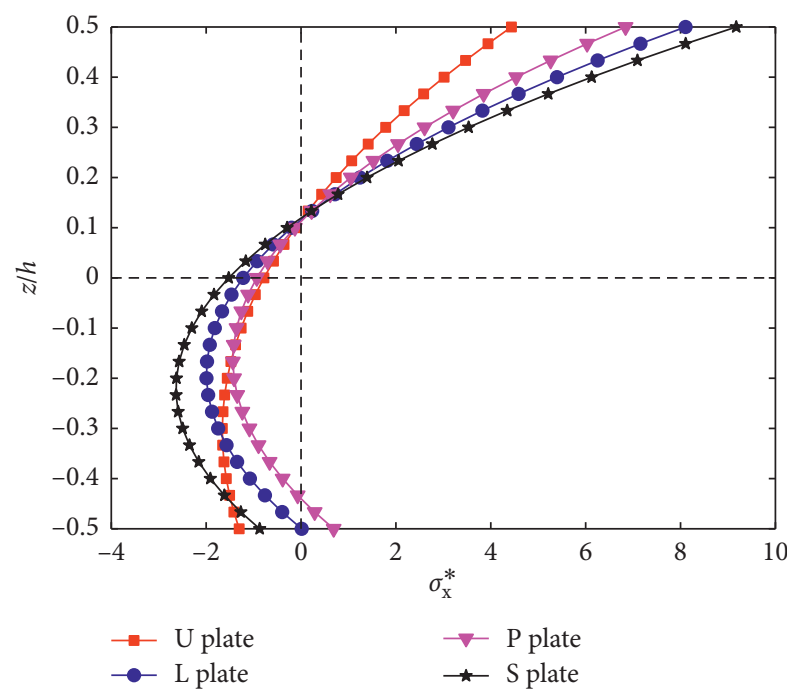

(a)

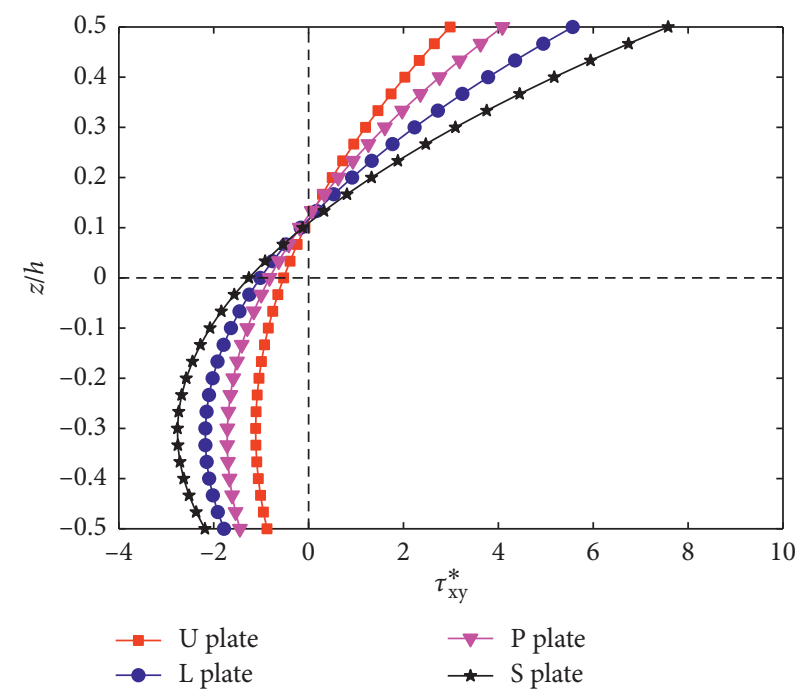

(b)

FIGURE 12: The distribution of normal and in-plane shear stresses through the thickness of the FGM plates. 


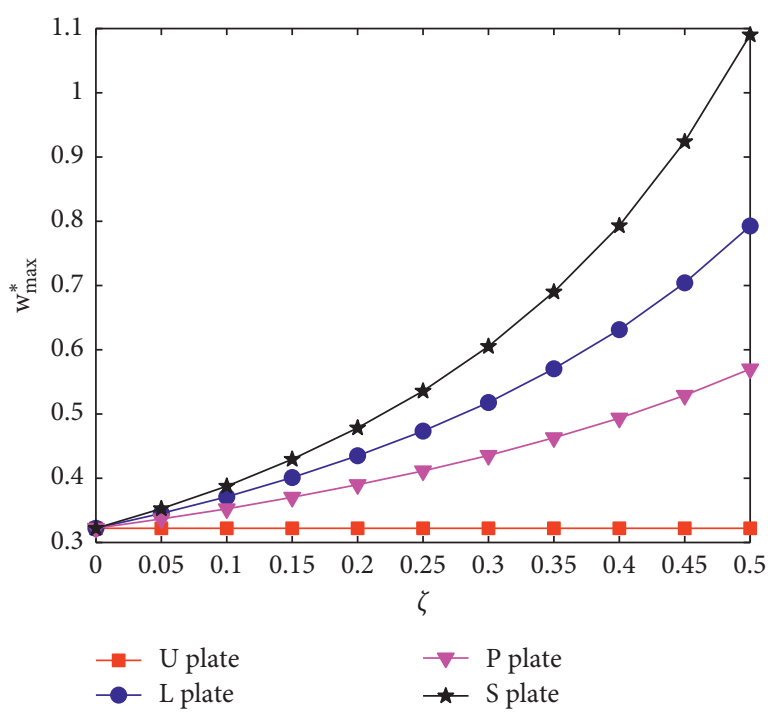

(a)

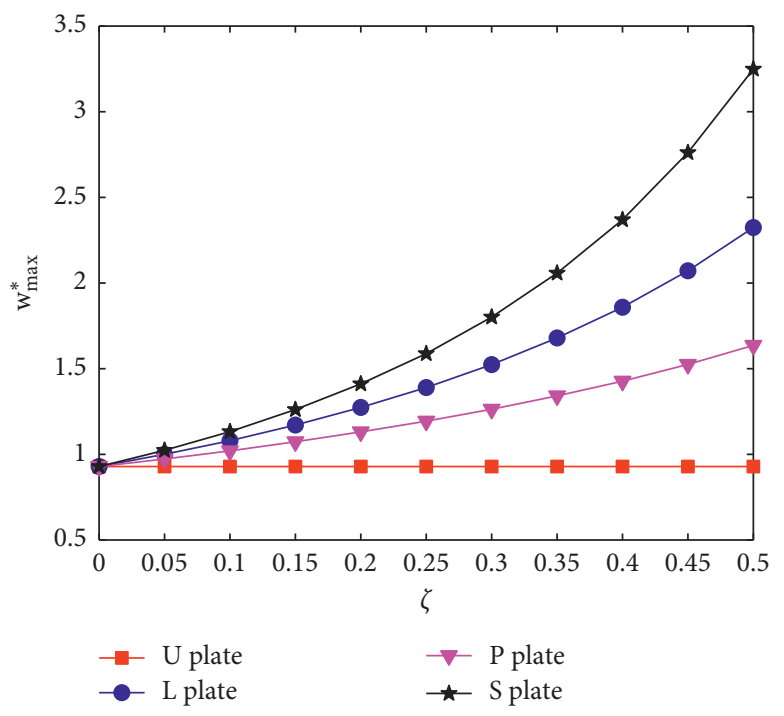

(c)

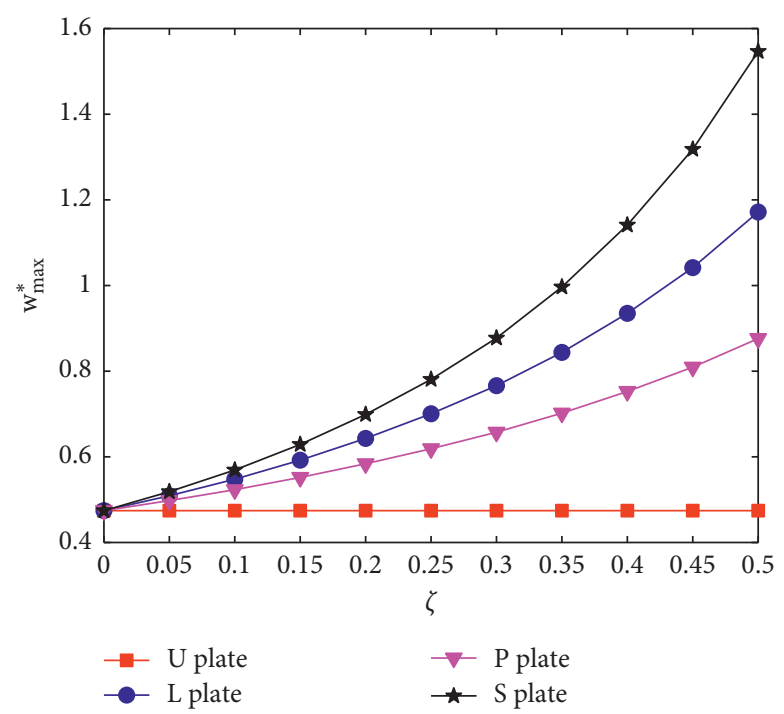

(b)

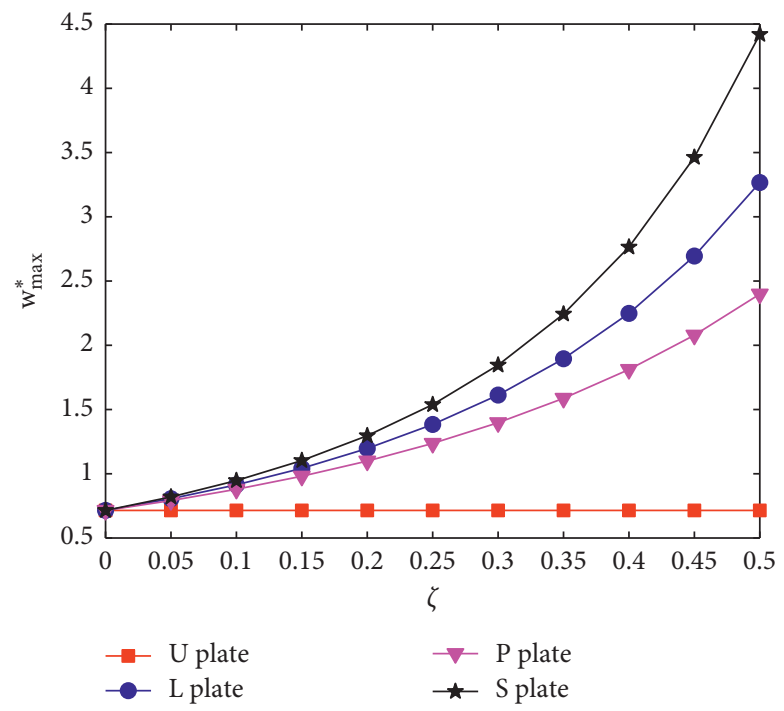

(d)

FIGURE 13: The influence of the thickness variation on the nondimensional maximum deflections of the FGM plates with different BCs: (a) CCCC plate, (b) SCSC plate, (c) SSSS plate, and (d) CFCF plate.

deflections of the CFCF plates are greatest while the CCCC plates are smallest. Additionally, the increasing rate of deflection of the CFCF plate is fastest.

\section{Conclusion}

In this work, a new mixed four-node quadrilateral plate element named MiQ4 with only five DOFs per node has been developed based on the FSDT and the mixed finite element formulation. Several examples were given to demonstrate the accuracy, high convergence rate, and efficiency of the proposed element. Then the proposed element MiQ4 is applied to research the static bending behavior of the FGM plates with variable thickness. Based on the results of the presented work, some remarkable conclusions can be clarified as follows:

The proposed element MiQ4 utilizes the bilinear shape function which is simple and similar to that of the wellknown classical Q4 element and allows us to calculate easily the numerical integration.

The new plate element is of good accuracy, high convergence rate, and reliability, especially for thin and very thin plates, in competition with other available elements.

The new element is insensitive to the mesh distortion, so it can be used to examine arbitrary and complex geometrical plates; as a consequence, the computation cost of the meshing process is reduced. 
The proposed element is free of shear locking without employing selective or reduced integrations.

Some numerical examples with different power-law index, side-to-thickness ratio, BCs, and thickness variation on the bending behavior of FGM plates are investigated.

The results of this study suggest some future works such as investigating the complex geometrical plates, studying the free vibration and dynamic response of the FGM plates subjected to complex loading in the thermal environment, and improving the convergent rate of the presented element.

\section{Data Availability}

No data were used to support this study.

\section{Conflicts of Interest}

The author declares that there are no conflicts of interest.

\section{References}

[1] J. N. Reddy, "Analysis of functionally graded plates," International Journal for Numerical Methods in Engineering, vol. 47, no. 1-3, pp. 663-684, 2000.

[2] A. S. Rezaei, A. R. Saidi, M. Abrishamdari, and M. H. P. Mohammadi, "Natural frequencies of functionally graded plates with porosities via a simple four variable plate theory: an analytical approach," Thin-Walled Structures, vol. 120, pp. 366-377, 2017.

[3] M. R. Barati and H. Shahverdi, "Nonlinear vibration of nonlocal four-variable graded plates with porosities implementing homotopy perturbation and Hamiltonian methods," Acta Mechanica, vol. 229, no. 1, pp. 343-362, 2018.

[4] M. R. Barati, H. Shahverdi, and A. M. Zenkour, "Electromechanical vibration of smart piezoelectric FG plates with porosities according to a refined four-variable theory," $\mathrm{Me}$ chanics of Advanced Materials and Structures, vol. 24, no. 12, pp. 987-998, 2017.

[5] S. J. Kumar and M. Mohammad, "Application of shifted Chebyshev polynomial-based Rayleigh-Ritz method and Navier's technique for vibration analysis of a functionally graded porous beam embedded in Kerr foundation," Engineering with Computers, 2020.

[6] B. Akgöz and Ö. Civalek, "Buckling analysis of functionally graded microbeams based on the strain gradient theory," Acta Mechanica, vol. 224, no. 9, pp. 2185-2201, 2013.

[7] M. A. Hamed, R. M. Abo-bakr, S. A. Mohamed, and M. A. Eltaher, "Influence of axial load function and optimization on static stability of sandwich functionally graded beams with porous core," Engineering with Computers, 2020.

[8] H. N. Nguyen, T. T. Hong, P. V. Vinh, and D. V. Thom, "An efficient beam element based on Quasi-3D theory for static bending analysis of functionally graded beams," Materials, vol. 12, no. 13, p. 2198, 2019.

[9] A. Frikha, A. Hajlaoui, M. Wali, and F. Dammak, "A new higher order C mixed beam element for FGM beams analysis," Composites Part B: Engineering, vol. 106, pp. 181-189, 2016.

[10] V. Nam, P. Vinh, N. Chinh, D. Thom, and T. Hong, "A new beam model for simulation of the mechanical behaviour of variable thickness functionally graded material beams based on modified first order shear deformation theory," Materials, vol. 12, no. 3, p. 404, 2019.

[11] S. Sahmani, A. M. Fattahi, and N. A. Ahmed, "Analytical treatment on the nonlocal strain gradient vibrational response of postbuckled functionally graded porous micro/nanoplates reinforced with GPL," Engineering with Computers, 2019.

[12] A. M. Zenkour, "Benchmark trigonometric and 3-D elasticity solutions for an exponentially graded thick rectangular plate," Archive of Applied Mechanics, vol. 77, no. 4, pp. 197-214, 2007.

[13] J. L. Mantari and C. Guedes Soares, "Bending analysis of thick exponentially graded plates using a new trigonometric higher order shear deformation theory," Composite Structures, vol. 94, no. 6, pp. 1991-2000, 2012.

[14] T. D. Van, P. V. Vinh, and H. N. Nguyen, "On the development of refined plate theory for static bending behavior of functionally graded plates," Mathematical Problems in Engineering, vol. 2020, Article ID 2836763, 13 pages, 2020.

[15] V. Mahesh and H. Dineshkumar, "Nonlinear vibration of functionally graded magneto-electro-elastic higher order plates reinforced by CNTs using FEM," Engineering with Computers, 2020.

[16] M. Khiloun, A. A. Bousahla, A. Kaci, A. Bessaim, A. Tounsi, and S. R. Mahmoud, "Analytical modeling of bending and vibration of thick advanced composite plates using a fourvariable quasi 3D HSDT," Engineering with Computers, vol. 36, no. 3, pp. 807-821, 2019.

[17] H. N. Vu, H. N. Nguyen, V. V. Pham, N. K. Dang, V. T. Do, and V. M. Phung, "A new efficient modified first-order shear model for static bending and vibration behaviors of two-layer composite plate," Advances in Civil Engineering, vol. 2019, Article ID 6814367, 17 pages, 2019.

[18] H. N. Nguyen, T. T. Hong, P. V. Vinh, N. D. Quang, and D. V. Thom, "A refined simple first-order shear deformation theory for static bending and free vibration analysis of advanced composite plates," Materials, vol. 12, no. 15, p. 2385, 2019.

[19] E. Carrera, S. Brischetto, M. Cinefra, and M. Soave, "Effects of thickness stretching in functionally graded plates and shells," Composites Part B: Engineering, vol. 42, no. 2, pp. 123-133, 2011.

[20] H. Mallek, H. Jrad, M. Wali, and F. Dammak, "Nonlinear dynamic analysis of piezoelectric-bonded FG-CNTR composite structures using an improved FSDT theory," Engineering with Computers, 2019.

[21] Q.-H. Pham, T.-D. Pham, Q. V. Trinh, and D.-H. Phan, "Geometrically nonlinear analysis of functionally graded shells using an edge-based smoothed MITC3 (ES-MITC3) finite elements," Engineering with Computers, vol. 36, no. 3, pp. 1069-1082, 2020.

[22] D. Chen, S. Kitipornchai, and J. Yang, "Dynamic response and energy absorption of functionally graded porous structures," Materials \& Design, vol. 140, pp. 473-487, 2018.

[23] D. Wu, A. Liu, Y. Huang, Y. Huang, Y. Pi, and W. Gao, "Dynamic analysis of functionally graded porous structures through finite element analysis," Engineering Structures, vol. 165 , pp. 287-301, 2018.

[24] H.-T. Thai and D.-H. Choi, "A simple first-order shear deformation theory for the bending and free vibration analysis of functionally graded plates," Composite Structures, vol. 101, pp. 332-340, 2013.

[25] H.-T. Thai and S.-E. Kim, "A simple higher-order shear deformation theory for bending and free vibration analysis of 
functionally graded plates," Composite Structures, vol. 96, pp. 165-173, 2013.

[26] A. M. Zenkour, "Generalized shear deformation theory for bending analysis of functionally graded plates," Applied Mathematical Modelling, vol. 30, no. 1, pp. 67-84, 2006.

[27] B. Hadj, B. Rabia, and T. H. Daouadji, "Influence of the distribution shape of porosity on the bending FGM new plate model resting on elastic foundations," Structural Engineering and Mechanics (SEM), vol. 72, no. 1, pp. 61-70, 2019.

[28] A. M. A. Neves, A. J. M. Ferreira, E. Carrera et al., "A quasi-3D sinusoidal shear deformation theory for the static and free vibration analysis of functionally graded plates," Composites Part B: Engineering, vol. 43, no. 2, pp. 711-725, 2012.

[29] H.-T. Thai and S.-E. Kim, "A simple quasi-3D sinusoidal shear deformation theory for functionally graded plates," Composite Structures, vol. 99, pp. 172-180, 2013.

[30] Q. X. Lieu, S. Lee, J. Kang, and J. Lee, "Bending and free vibration analyses of in-plane bi-directional functionally graded plates with variable thickness using isogeometric analysis," Composite Structures, vol. 192, pp. 434-451, 2018.

[31] B. Temel and A. R. Noori, "A unified solution for the vibration analysis of two-directional functionally graded axisymmetric Mindlin-Reissner plates with variable thickness," International Journal of Mechanical Sciences, vol. 174, Article ID 105471, 2020.

[32] O. C. Zienkiewicz, R. L. Taylor, and J. M. Too, "Reduced integration technique in general analysis of plates and shells," International Journal for Numerical Methods in Engineering, vol. 3, no. 2, pp. 275-290, 1971.

[33] T. J. R. Hughes, R. L. Taylor, and W. Kanoknukulchai, "A simple and efficient finite element for plate bending," International Journal for Numerical Methods in Engineering, vol. 11, no. 10, pp. 1529-1543, 1977.

[34] E. Hinton and H. C. Huang, "A family of quadrilateral Mindlin plate elements with substitute shear strain fields," Computers \& Structures, vol. 23, no. 3, pp. 409-431, 1986.

[35] K.-J. Bathe and E. N. Dvorkin, "A four-node plate bending element based on Mindlin/Reissner plate theory and a mixed interpolation," International Journal for Numerical Methods in Engineering, vol. 21, no. 2, pp. 367-383, 1985.

[36] J. L. Batoz and P. Lardeur, "A discrete shear triangular nine D.O.F. element for the analysis of thick to very thin plates," International Journal for Numerical Methods in Engineering, vol. 28, no. 3, pp. 533-560, 1989.

[37] O. C. Zienkiewicz, Z. Xu, L. F. Zeng, A. Samuelsson, and N.-E. Wiberg, "Linked interpolation for Reissner-Mindlin plate elements: Part I-A simple quadrilateral," International Journal for Numerical Methods in Engineering, vol. 36, no. 18, pp. 3043-3056, 1993.

[38] D. Ribaric and G. Jelenic, "Higher-order linked interpolation in quadrilateral thick plate finite elements," Finite Elements in Analysis and Design, vol. 51, pp. 67-80, 2012.

[39] A. K. Soh, Z. F. Long, and S. Cen, "A new nine DOF triangular element for analysis of thick and thin plates," Computational Mechanics, vol. 24, no. 5, pp. 408-417, 1999.

[40] A.-K. Soh, S. Cen, Y.-Q. Long, and Z.-F. Long, "A new twelve DOF quadrilateral element for analysis of thick and thin plates," European Journal of Mechanics - A/Solids, vol. 20, no. 2, pp. 299-326, 2001.

[41] G. Castellazzi and P. Krysl, "A nine-node displacement-based finite element for Reissner-Mindlin plates based on an improved formulation of the NIPE approach," Finite Elements in Analysis and Design, vol. 58, pp. 31-43, 2012.
[42] P. Hansbo, D. Heintz, and M. G. Larson, "A finite element method with discontinuous rotations for the MindlinReissner plate model," Computer Methods in Applied Mechanics and Engineering, vol. 200, no. 5-8, pp. 638-648, 2011.

[43] P. Hansbo and M. G. Larson, "Locking free quadrilateral continuous/discontinuous finite element methods for the Reissner-Mindlin plate," Computer Methods in Applied Mechanics and Engineering, vol. 269, pp. 381-393, 2014.

[44] R. Ayad, G. Dhatt, and J. L. Batoz, "A new hybrid-mixed variational approach for Reissner-Mindlin plates. The MiSP Model," International Journal for Numerical Methods in Engineering, vol. 42, no. 7, pp. 1149-1179, 1998.

[45] R. Ayad and A. Rigolot, "An improved four-node hybridmixed element based upon Mindlin's plate theory," International Journal for Numerical Methods in Engineering, vol. 55, no. 6, pp. 705-731, 2002.

[46] N. Nguyen-Thanh, T. Rabczuk, H. Nguyen-Xuan, and S. Bordas, "An alternative alpha finite element method with discrete shear gap technique for analysis of isotropic MindlinReissner plates," Finite Elements in Analysis and Design, vol. 47, no. 5, pp. 519-535, 2011.

[47] G. R. Liu and T. Nguyen-Thoi, Smoothed Finite Element Methods, CRC Press, Boca Raton, FL, USA, 2010.

[48] G. R. Liu, T. T. Nguyen, K. Y. Dai, and K. Y. Lam, "Theoretical aspects of the smoothed finite element method (SFEM)," International Journal for Numerical Methods in Engineering, vol. 71, no. 8, pp. 902-930, 2007.

[49] H. Nguyen-Xuan and T. Nguyen-Thoi, "A stabilized smoothed finite element method for free vibration analysis of Mindlin-Reissner plates," Communications in Numerical Methods in Engineering, vol. 25, no. 8, pp. 882-906, 2009.

[50] T. Nguyen-Thoi, P. Phung-Van, H. Luong-Van, H. NguyenVan, and H. Nguyen-Xuan, "A cell-based smoothed threenode Mindlin plate element (CS-MIN3) for static and free vibration analyses of plates," Computational Mechanics, vol. 51, no. 1, pp. 65-81, 2013.

[51] T. Nguyen-Thoi, T. Rabczuk, V. Ho-Huu, L. Le-Anh, H. Dang-Trung, and T. Vo-Duy, "An extended cell-based smoothed three-node mindlin plate element (XCS-MIN3) for free vibration analysis of cracked FGM plates," International Journal of Computational Methods, vol. 14, no. 2, p. 1750011, 2017.

[52] H. Nguyen-Xuan, G. R. Liu, C. Thai-Hoang, and T. NguyenThoi, "An edge-based smoothed finite element method with stabilized discrete shear gap technique for analysis of Reissner-Mindlin plates," Computer Methods in Applied Mechanics and Engineering, vol. 199, pp. 471-489, 2009.

[53] T. Nguyen-Thoi, G. R. Liu, H. Nguyen-Xuan, and C. NguyenTran, "Adaptive analysis using the node-based smoothed finite element method (NS-FEM)," Communications in $\mathrm{Nu}$ merical Methods in Engineering, vol. 27, no. 2, pp. 198-218, 2009.

[54] Y. Q. Long and K. G. Xin, "Generalized conforming element for bending and buckling analysis of plates," Finite Elements in Analysis and Design, vol. 5, no. 1, pp. 15-30, 1989.

[55] Y. Long and Y. Xu, "Generalized conforming quadrilateral membrane element with vertex rigid rotational freedom," Computers \& Structures, vol. 52, no. 4, pp. 749-755, 1994.

[56] Y. Q. Long and Y. Xu, "Generalized conforming triangular membrane element with vertex rigid rotational freedoms," Finite Elements in Analysis and Design, vol. 17, no. 4, pp. 259-271, 1994.

[57] Y. Q. Long, X. M. Bu, Z. F. Long, and Y. Xu, "Generalized conforming plate bending elements using point and line 
compatibility conditions," Computers \& Structures, vol. 54, no. 4, pp. 717-723, 1995.

[58] S. Cen, Y.-Q. Long, Z.-H. Yao, and S.-P. Chiew, "Application of the quadrilateral area co-ordinate method: a new element for Mindlin-Reissner plate," International Journal for $\mathrm{Nu}$ merical Methods in Engineering, vol. 66, no. 1, pp. 1-45, 2006.

[59] S. Cen, Y. Shang, C.-F. Li, and H.-G. Li, "Hybrid displacement function element method: a simple hybrid-Trefftz stress element method for analysis of Mindlin-Reissner plate," International Journal for Numerical Methods in Engineering, vol. 98, no. 3, pp. 203-234, 2014.

[60] J. Jirousek, A. Wróblewski, Q. H. Qin, and X. Q. He, “A family of quadrilateral hybrid-Trefftz p-elements for thick plate analysis," Computer Methods in Applied Mechanics and Engineering, vol. 127, no. 1-4, pp. 315-344, 1995.

[61] I. Katili, "A new discrete Kirchhoff-Mindlin element based on Mindlin-Reissner plate theory and assumed shear strain fields-Part II: an extended DKQ element for thick-plate bending analysis," International Journal for Numerical Methods in Engineering, vol. 36, no. 11, pp. 1885-1908, 1993.

[62] T. J. R. Hughes, The Finite Element Method: Linear Static and Dynamic Finite Element Analysis, Dover Publications, Mineola, NY, USA, 2000.

[63] L. S. D. Morley, Skew Plates and Structures, Pergamon Press, Oxford, UK, 1963. 